\title{
A Mathematical Foundation for Foundation Paper Pieceable Quilts
}

\author{
MACKENZIE LEAKE, Stanford University, USA \\ GILBERT BERNSTEIN, UC Berkeley, USA \\ ABE DAVIS, Cornell University, USA \\ MANEESH AGRAWALA, Stanford University, USA
}

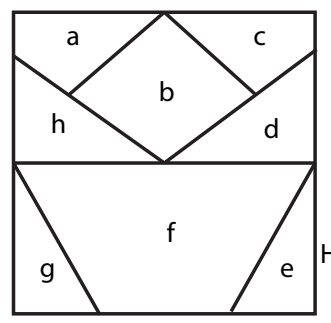

Input pattern design

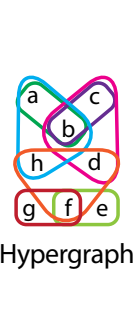

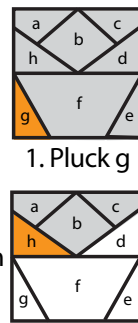

5. Pluck h
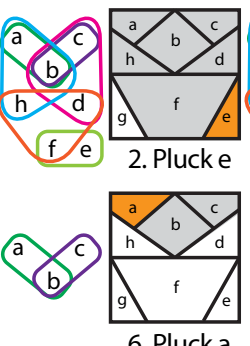

6. Pluck a

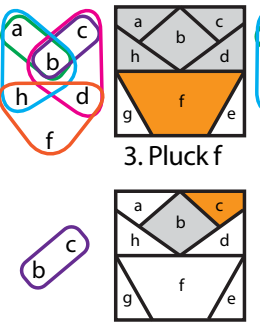

7. Pluck c

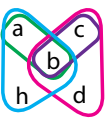

b
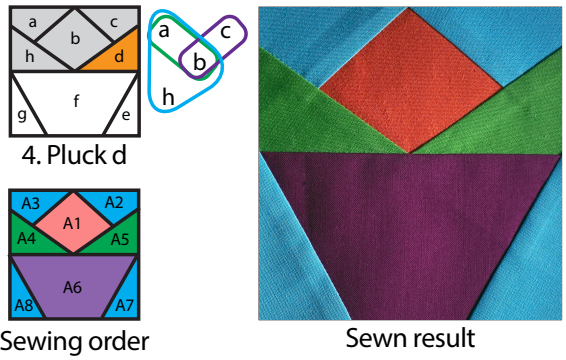

Sewn result

Fig. 1. Given an input pattern design we encode the geometry as a dual hypergraph, where nodes represent faces and hyperedges represent seams connecting two or more faces. We visualize the hyperedges with colored boundaries (left). In this work we prove that if this hypergraph is acyclic, the pattern design is foundation paper pieceable, and we present a leaf-plucking algorithm that iteratively removes leaf hyperedges, where a node is only contained in that hyperedge, to generate a sewing order for the design, which is the reverse of the order in which we plucked the nodes (center). Our quilt design tool shows the resulting sewing order by numbering the faces (center, Sewing order) and lets users color the faces to visualize the design. Quilters can use foundation paper piecing to sew the quilt by attaching fabric pieces one at a time in the sewing order and precisely construct the quilt top (right).

Foundation paper piecing is a popular technique for constructing fabric patchwork quilts using printed paper patterns. But, the construction process imposes constraints on the geometry of the pattern and the order in which the fabric pieces are attached to the quilt. Manually designing foundation paper pieceable patterns that meet all of these constraints is challenging. In this work we mathematically formalize the foundation paper piecing process and use this formalization to develop an algorithm that can automatically check if an input pattern geometry is foundation paper pieceable. Our key insight is that we can represent the geometric pattern design using a certain type of dual hypergraph where nodes represent faces and hyperedges represent seams connecting two or more nodes. We show that determining whether the pattern is paper pieceable is equivalent to checking whether this hypergraph is acyclic, and if it is acyclic, we can apply a leaf-plucking algorithm to the hypergraph to generate viable sewing orders for the pattern geometry. We implement this algorithm in a design tool that allows quilt designers to focus on producing the geometric design of their pattern and let the tool handle the tedious task of determining whether the pattern is foundation paper pieceable.

CCS Concepts: • Computing methodologies $\rightarrow$ Graphics systems and interfaces.

Additional Key Words and Phrases: quilting, design tools, craft, fabrication

Authors' addresses: Mackenzie Leake, Stanford University, Stanford, California USA, mleake@cs.stanford.edu; Gilbert Bernstein, UC Berkeley, Berkeley, California, USA, gilbo@berkeley.edu; Abe Davis, Cornell University, Ithaca, New York, USA abedavis@cornell.edu; Maneesh Agrawala, Stanford University, Stanford, California, USA, maneesh@cs.stanford.edu.

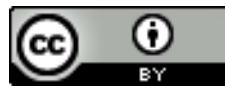

This work is licensed under a Creative Commons Attribution International 4.0 License. (c) 2021 Copyright held by the owner/author(s).

0730-0301/2021/8-ART65

https://doi.org/10.1145/3450626.3459853

\section{ACM Reference Format:}

Mackenzie Leake, Gilbert Bernstein, Abe Davis, and Maneesh Agrawala. 2021. A Mathematical Foundation for Foundation Paper Pieceable Quilts. ACM Trans. Graph. 40, 4, Article 65 (August 2021), 14 pages. https://doi.org/ $10.1145 / 3450626.3459853$

\section{INTRODUCTION}

Quiltmaking has become a popular craft, with 7-10 million quilters in the US alone [The Quilting Company 2017]. Foundation paper piecing is a common method for sewing the top layer of a quilt using a pattern printed on paper as a physical guide. Quilters sew pieces of fabric corresponding to each polygon in the pattern's geometric design along the printed seam lines, directly to the paper and one another. The fabric pieces are sewn one at a time in the sewing order specified by the numbering of the polygons (Figure 1). After sewing all interior seams in the pattern, quilters remove the paper, resulting in a precise fabric patchwork quilt top ready to be layered atop batting material and backing fabric, and then sewn together into a finished quilt.

The paper guide serves as a foundation for the construction process that provides stability and increases precision compared to traditional quilt piecing techniques that do not use paper [Alteneder 2020; Mahoney 2016; Sharp 2018]. Specifically, it provides a stable base for aligning the fabric pieces and holding them in place via pins during sewing. The printed seam lines serve as precise visual guides for sewing the seams accurately. Sewing a straight seam at a specific location is far more difficult when there is no printed line to follow along. These advantages of paper piecing have made it a widely used method for sewing quilt tops, especially among beginners, but also for many experienced quilters [Mahoney 2016]. 


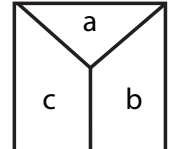

(A) Y-junction $*$ (B) Mondrian $*$

\begin{tabular}{|c|c|c|c|}
\hline a & & $\mathrm{a}$ & $b$ \\
\hline \multirow{2}{*}{$d$} & \multirow{2}{*}{ C } & \multicolumn{2}{|c|}{$C$} \\
\hline & & & $d$ \\
\hline
\end{tabular}

(C) Separated Mondrian $*$

\begin{tabular}{|l|l|l|}
\hline \multicolumn{2}{|c|}{$a$} & \\
\hline$d$ & $e$ & $b$ \\
\cline { 2 - 3 } & & \\
\cline { 2 - 3 } & & \\
\hline
\end{tabular}

(D) Rose $*$

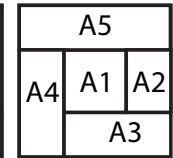

(E) Log cabin $\checkmark$
Fig. 2. Many geometric patterns designs, such as the Y-junction (A), Mondrian (B), Separated Mondrian (C), and Rose (D), are not paper pieceable. Although the Log Cabin (E) is geometrically similar to the Rose (D), it is paper pieceable in the order given by its numbering.

However, designing a foundation paper pieceable pattern is challenging because the construction process imposes a variety of constraints on the geometry and sewing order. For example, one well known constraint on the geometry is that all intersections between seams must form T-junctions [Sharp 2018]. If a pattern contains a junction that does not form a T, it cannot be sewn using foundation paper piecing. But the converse is not true; a pattern geometry can contain only $\mathrm{T}$-junctions and still be impossible to construct via foundation paper piecing (Figure 2B-D). Unfortunately no complete set of such constraints-that would allow a quilt designer to easily check if their own pattern design is paper pieceable-is known.

Instead, books, websites, and courses on designing foundation paper pieceable patterns [Kerns 2020; Sharp 2018; Worland 2020] suggest iterating between designing the geometry, manually checking that a viable sewing order exists for it, and then redesigning the geometry if no such order exists. But, manually identifying a viable sewing order is tedious and error prone. The designer must consider many different orderings, and mentally walk through the paper piecing construction process for each one to check whether sewing the fabric pieces in that order is physically feasible. As pattern geometry becomes more complex (i.e., contains more faces and seams), checking for a viable sewing order becomes more difficult, and designers often end up checking only a few possible orderings before giving up and choosing to redesign the geometry.

In this paper, we present a design tool that can automatically check if an input geometric design is foundation paper pieceable. To build this tool, we first develop a mathematical formalization of the foundation paper piecing process. The key idea of our formalization is to represent the geometric pattern design as a certain type of dual hypergraph where nodes represent faces and hyperedges represent seams connecting two of more nodes. By treating the sewing order as a sequence of transitions in a finite state machine, we show that determining whether the geometric design is foundation paper pieceable is equivalent to checking whether the dual hypergraph is acyclic. Moreover, if it is acyclic, we can use a leaf-plucking algorithm to generate all viable sewing orders for the design. We show that the resulting algorithm effectively computes sewing orders in reverse; starting from the complete input pattern, it iteratively removes a face as long as it can be separated from the pattern by cutting along a single seam line. It is efficient enough to provide real-time feedback showing whether or not the design is paper pieceable as a quilter is designing the geometry. By eliminating the tedious manual step of determining whether a pattern is foundation paper pieceable, our tool allows quilters to focus on the visual design of the pattern rather than the sewing order.

\section{RELATED WORK}

Researchers have developed digital design tools for fabrication, focusing on a wide variety of different processes and materials including knitting with yarn [McCann et al. 2016; Narayanan et al. 2018], shaping inflatable plastic balloons [Furuta et al. 2010; Skouras et al. 2012, 2014], cutting and folding paper [Demaine and Demaine 2002; Kilian et al. 2008; Mitani and Suzuki 2004], designing interlocking joints for furniture [Larsson et al. 2020; Yao et al. 2017], and sewing cloth to make garments [Bartle et al. 2016; Berthouzoz et al. 2013; Igarashi and Hughes 2002; Umetani et al. 2011; Wolff and Sorkine-Hornung 2019] and other soft objects [Mori and Igarashi 2007]. Bickel et al.'s [2018] recent survey covers many of these fabrication design tools in detail. One particularly relevant class of such tools focuses on generating scaffolds and jigs to aid the fabrication process. For example, Holly [Igarashi and Igarashi 2010] is a tool for designing stencils that can be painted through to produce graphic artwork. WrapIt [Iarussi et al. 2015] and ProxyPrint [Torres et al. 2016] are tools for 3D printing customized jigs to produce wrapped wire jewelry. Deuss et al. [2014] design chain-based scaffolds for assembling self-supporting masonry models, while Garg et al. [2014] generate laser cut wooden scaffolds for fabricating 3D wire meshes. The physical paper guide in foundation paper piecing similarly serves as a scaffold for the construction process, and our work is aimed at facilitating the design of these paper guides.

Closest to our work are tools aimed at generating quilting patterns. A few research groups have developed techniques for producing free motion quilt patterns, where continuous stitching is used as a primary design element for stitching through the layers of a quilt. Some of these techniques create the stitching patterns using procedural curve generation techniques [Carlson et al. 2015; Li et al. 2019]. Liu et al. [2017] work from an underlying photograph and combine edge extraction with continuous line generation techniques to produce the stitching curve. Patchy [Igarashi and Mitani 2015] lets users visualize the fabric pieces in a pieced pattern as well as the stitching between them. Coahran and Fiume [2005] develop a tool for designing Bargello quilts which are formed by parallel rows of colored curves composed solely of rectangular fabric pieces. Foundry [Smith 2017] is a parameterized tool for generating and visualizing foundation paper pieceable quilts. It uses a template-based approach based on binary space partitions to generate the patterns, but details of the method have not yet been published.

Commercial software tools for designing pieced quilts focus on letting users draw and color geometric block patterns and arrange the blocks to form a quilt [Cosman 2012; ElectricQuilt 2017; PreQuilt 2020; Quiltster 2020]. A few of these tools can break input pattern geometry into multiple foundation paper pieceable sections. But because the tools are proprietary, it is unclear how robustly they can identify paper pieceable sections for all input geometries. In contrast, we focus on mathematically formalizing the foundation paper piecing process to develop a robust algorithm for identifying when an input pattern geometry is paper pieceable. To our knowledge we are the first to give a mathematically precise definition of paper pieceability and provide a provably complete algorithm for producing all viable sewing orders for a given pattern geometry. 
Foundation Paper Piecing Construction Process
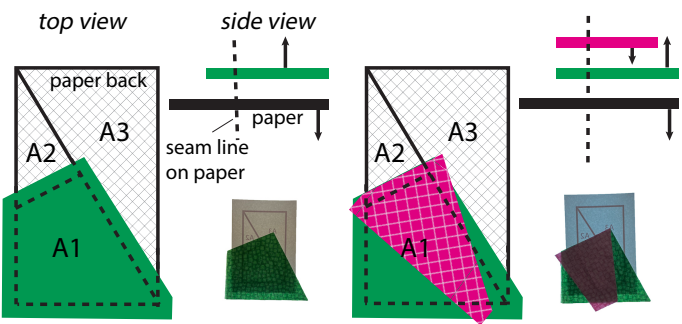

(1) Layer: Lay fabric 1 over A1 right-side-up. Lay fabric 2 wrong-side-up along the seam between $\mathrm{A} 1$ \& $\mathrm{A} 2$.

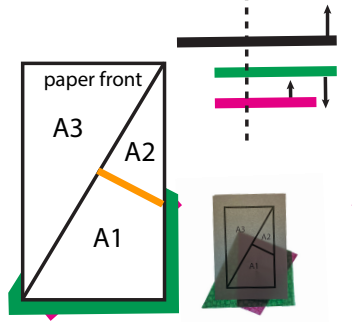

(2) Stitch: Flip the stack over so that the paper is on top. Stitch the seam between $\mathrm{A} 1$ \& A2 from the paper side.
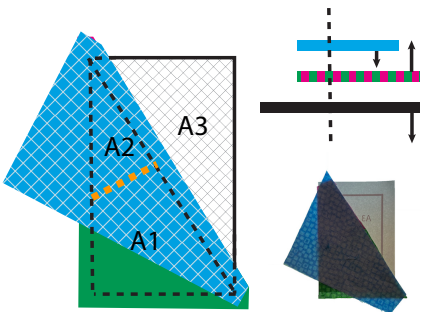

(1) Layer: Lay fabric 3 wrong-side-up along seam between $\mathrm{A} 1 / \mathrm{A} 2$ \& $\mathrm{A} 3$.
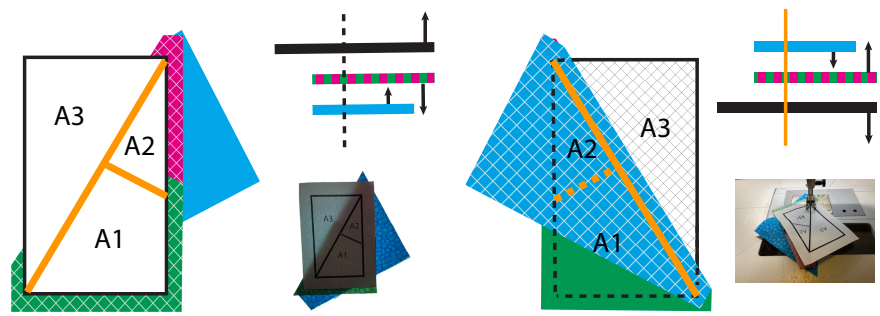

(2) Stitch: Flip the stack over so that the paper is on top. Stitch the seam between $A 1 / A 2 \& A 3$ from the paper side.

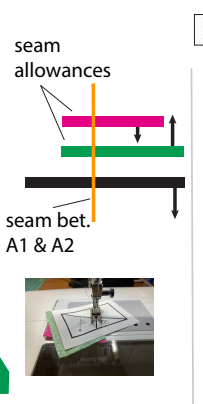

(3) Fold: Flip the stack. Fold and press fabric 2 to cover A2.

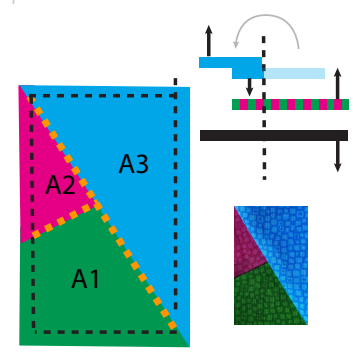

(3) Fold: Flip the stack. Fold and press fabric 3 to cover A3.

Fig. 3. Constructing a section of a foundation paper pieceable pattern starts by sewing the first two fabric pieces (top row) which involves three main steps. In the layering step the quilter places the paper pattern wrong-side-up (printed side down) and pins the fabric piece 1 right-side-up, centered on face A1 of the paper so that its seam allowances extend outside the face on all sides. The quilter then pins fabric 2 , wrong-side-up, on top of fabric 1 so that the seam allowances for both fabrics then extend outside face A1 and into face A2. In the stitching step the quilter flips over the layered stack and sews along the printed seam line, attaching fabrics 1 and 2 to the paper and one another, and then flips the stack back over. Finally, in the folding step the quilter folds fabric 2 over its seam allowance and presses it into position so that both fabrics are right-side-up and both seam allowances as well as the seam stitching are hidden underneath fabric 2. Each subsequent fabric is similarly sewn into the quilt, one at a time in the sewing order given by the pattern, using these three steps (bottom row). In this case, fabric 3 is sewn to both fabrics 1 and 2 when it is attached.

\section{FOUNDATION PAPER PIECING}

We have analyzed a variety of books, websites and courses on foundation paper piecing [Alteneder 2020; Designs 2020; Grzych 2018; Kerns 2020; Mahoney 2016; Sharp 2018; Worland 2020] and found that most of them focus on describing the construction process. We briefly summarize this process in Section 3.1. None of these resources describes a complete set of higher-level geometric constraints for a pattern geometry to be foundation paper pieceable. However, in analyzing these resources, we have identified a set of three key requirements of the foundation paper piecing construction process. In Section 3.2 we describe these requirements, and then in Section 3.3 we discuss how manually checking them makes it challenging to design foundation paper pieceable patterns.

\subsection{Construction Process}

A foundation paper piecing quilt pattern describes both the geometric design of the quilt top as well as the sewing order in which faces should be sewn into the quilt (inset). Each polygon (or face) in the design represents a

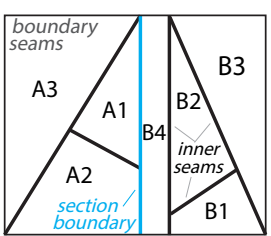
different piece of fabric, the letters divide the design into sections that can be constructed using a single piece of paper as a physical guide, and for each section the numbers describe the order in which the faces should be sewn into the pattern. The inset contains two sections, $\mathrm{A}$ and $\mathrm{B}$.

To construct a single section of a foundation paper pieceable pattern, a quilter initially prints the section geometry on physical paper and cuts a piece of fabric for each numbered face, large enough to both cover the face and extend by a one-quarter inch seam allowance beyond each of its edges. Figure 3 illustrates the process of sewing each piece of fabric into the quilt, according to the sewing order. This process involves three main steps (1) layering the new fabric piece wrong-side-up atop the already attached fabric pieces that are lying right-side-up, with the paper pattern underneath, lying wrong-side up, (2) stitching the paper pattern and fabric pieces together along a seam, and then (3) folding the new fabric piece into position so that it lies right side up and hides the seam. The section is complete when each of the fabric pieces has been added to the quilt and all interior/inner seams have been sewn.

After sewing each section of the quilt independently using this paper piecing process, the quilter typically joins the sections together at their boundaries using traditional seam sewing methods (i.e., without using a paper guide). Since traditional seam sewing does not impose any design constraints on the section boundaries, we focus in this work on single section foundation paper piecing. 


\subsection{Requirements of Foundation Paper Piecing}

We have observed three requirements of the foundation paper piecing process that allow the technique to hide seams while providing stability and precision advantages over traditional quilt piecing methods that do not use paper.

(1) Layering. Before sewing a seam, the paper and fabric must be layered together as follows: First, the paper lies wrong-side-up as the foundation; Second, fabric on one side of the seam must lie rightside-up on top of the paper; Third, the fabric that is ultimately folded to lie on the opposite side of the seam must be initially layered wrong-side-up on the same side of the seam as the second layer. This configuration ensures that the stitching and seam allowances are hidden behind the wrong-side of the resulting quilt top.

(2) Flatness. When stitching a seam, the paper and fabrics must all lie flat at the seam. This requirement ensures that quilters can easily sew through exactly three layers without interference from other parts of the paper and thereby allows quilters to precisely construct a stable and high-quality quilt top.

(3) No Refolding. After sewing the wrong-side-up piece of fabric, it is folded to lie right-side-up in its desired position. From this point on, it must remain right-side-up and flat against the paper. In particular, it cannot be used as the wrong-side-up fabric when sewing another seam, because that would require refolding the fabric and violate our flatness requirement.

Consider the geometric design in Figure 3. Earlier we saw that it is foundation paper pieceable as we can sew it by attaching the pieces in the order (A1, A2, A3), and never violate any of our requirements. In contrast, consider the order (A3, A2, A1). We first sew the seam between $\mathrm{A} 3$ and $\mathrm{A} 2$ and then add $\mathrm{A} 1$ by sewing its seam with $\mathrm{A} 3$. Now we cannot sew the remaining seam between $A 1$ and $A 2$ because they are both already attached to the paper. There is no way to orient either A1 or A2 wrong-side-up without violating the no refolding requirement. This sewing order is not foundation paper pieceable.

So when can a seam be sewn successfully? The fabric along at least one side of the seam must be unattached from the paper, or else we cannot layer it wrong-side-up. This leaves us with two basic scenarios: either the fabric pieces on both sides of the seam are unattached, or a single piece of fabric is unattached, and all of the fabric pieces along the opposite side of the seam are attached Following this intuition, we will later define Type- 0 and Type- 1 sewing operations (Definition 4.7). Type-0 operations attach two previously unattached pieces of fabric-which is always and only the first seam to be sewn. Type- 1 operations attach a piece of fabric on one side of a seam to the fabric already present on the other side.

We can also observe pattern designs for which it is impossible to find any foundation paper pieceable sewing order and identify corresponding requirements that pieceable designs must meet. For instance, Y-junctions (Figure 2A) are never paper pieceable. (Observe that all possible orders are symmetric and try to work through the construction process; you'll run into a violation of one of our requirements.) Even when we restrict the pattern to contain only T-junctions, there remain many designs for which none of the possible sewing orders works (Figure 2B-D). In Section 4 we develop a geometric theory that extends and systematizes our intuitive grasp of when a pattern is or is not foundation paper pieceable.

\subsection{Designing Foundation Paper Pieceable Patterns}

The current practice of designing a foundation paper piecing pattern involves iterating between designing the geometry, manually checking that a viable sewing order exists for it, and then either redesigning the geometry or breaking the design into multiple independent sections if no such ordering exists [Kerns 2020; Sharp 2018; Worland 2020]. Manually identifying a viable sewing order is especially challenging because the designer must usually consider many different orderings and mentally walk through the foundation paper piecing procedure (Section 3.1) for each one, to check whether any of the requirements (Section 3.2) are violated. With complex designs containing many faces and seams, designers often end up checking only a few of the possible orderings because the procedure is mentally taxing, and decide to break the pattern into multiple sections rather than continuing to look for a viable sewing order. In some cases designers mistakenly determine that a sewing order is viable only to find later on as they are part way through constructing the pattern that the ordering does not work. Correcting such errors can be very costly in terms of time and materials. Our work formalizes the foundation paper piecing process and automates the check for viable sewing orders so that quilt designers can focus their effort on developing the geometry of the pattern. Our algorithm suggests a new rule-of-thumb for determining a valid foundation paper pieceable sewing order for an input pattern: start from the complete pattern and iteratively remove a face as long as it can be separated from the pattern by cutting along a single seam line.

\section{THEORY}

In order to formalize the foundation paper piecing process, we first define a quilt design as a planar mesh whose edges may be grouped into seams in different ways (Section 4.1). We define the construction process itself as reachability in a state transition system, whose transitions are basic sewing operations (Section 4.2), and show certain necessary properties of foundation paper pieceable designs (Section 4.3). However, these properties are not sufficient to guarantee paper pieceability, so finally we define the dual-hypergraph of a design (Section 4.4) and prove that a given design is foundation paper pieceable if and only if its dual hypergraph is acyclic (Section 4.5). Note that while we present the intuition for the theorems and many of the lemmas in this Theory Section, we provide detailed proofs for them in Appendix A.

\subsection{Designs and Seams}

Intuitively, we will define a design as a planar mesh, without any order specified on the constituent polygonal faces/pieces. More precisely, we define a design as follows.

Definition 4.1 (Designs). Let $G=(V, F, x)$ be a planar mesh where $V$ is the set of vertices, $F$ is the set of faces (each specified as a cyclically ordered list of vertices) and $x: V \rightarrow \mathbb{R}^{2}$ is the positions of vertices. Every planar mesh implicitly defines a set of edges $E$, each of which is a pair of subsequent vertices in some face. 
In this work, we make two assumptions of every planar mesh. (1) They must be non-degenerate (no repeated vertices in faces, no vertices outside of faces, no coincident vertices, no intersecting edges and no overlapping faces). (2) There are no degree 2 vertices whose incident edges are colinear.

Additionally, recall that the realization of a mesh is the set of points in the plane lying on a face, edge, or vertex of the mesh. The boundary and interior of a mesh is simply the boundary and interior of its realization. In this sense, we can talk about whether vertices/edges of the mesh are boundary or interior vertices/edges A Design is a planar mesh $G$ whose interior is connected.

Definition 4.2 (Sub-Designs). A sub-design $G^{\prime}=\left(V^{\prime}, F^{\prime}, x^{\prime}\right)$ of a design $G=(V, F, x)$ is induced by choosing a subset of faces $F^{\prime} \subseteq F$. $V^{\prime}$ is the set of vertices occurring in $F^{\prime}$ and $x^{\prime}$ is the restriction of $x$ to $V^{\prime}$. Note that a subset of faces $F^{\prime}$ only specifies a sub-design if $G^{\prime}$ is a design, which is not true if $G^{\prime}$ is not connected.

In a given design, there are multiple seams: contiguous and colinear runs of edges that can potentially be sewn together in a single sewing operation. As discussed in Section 3.2, when sewing a seam, a single piece of fabric is attached along one side of the seam. To capture this property in our theory, we require (by definition) that the edges of a seam always share such a common face. For example in Figure 4A, we see that the sequence $(e 1, e 2)$ is a seam with common face $F 1$, but that $(e 1, e 2, e 3)$ is not a seam because these edges do not share a single common face.

Definition 4.3 (Seam). Given a design $G=(V, F, x)$ with interior edges $E$, a seam $s=\left(e_{1}, \ldots, e_{k}\right)$ is a sequence of one or more interior edges, such that (1) each subsequent pair of edges $e_{i}, e_{i+1}$ is contiguous and colinear; (2) the shared vertex between each pair lies in the interior of the mesh; and (3) all of the edges are adjacent to a common face. We say that a seam is simple if it consists of a single edge (all internal edges of a design are simple seams) and that a seam is complex if it consists of more than one edge.

By this definition, seams for the pattern geometry in Figure $4 \mathrm{~A}$ include $s_{1}=(e 1), s_{2}=(e 2), s_{3}=(e 3), s_{4}=(e 4), s_{5}=(e 1, e 2), s_{6}=$ $(e 2, e 3)$, and $s_{7}=(e 3, e 4)$. The first four are simple seams and the last three are complex seams. The contiguous and colinear sequence of edges $(e 1, e 2, e 3, e 4)$ is not a seam because there is no single face that is adjacent to all four of these edges. Furthermore, $s_{1}, \ldots s_{4}$ are all sub-sequences of one or more complex seams. We capture this sub-sequence relationship, with two additional definitions.

Definition 4.4 (Sub-Seam). A seam $s_{i}$ is a sub-seam of a seam $s_{j}$ $\left(s_{i} \leq s_{j}\right)$ iff. $s_{i}$ is a sub-sequence of $s_{j}$.

Definition 4.5 (Maximal Seams). A maximal seam is a seam that is not a sub-seam of some other seam.

One of our main results is to prove that for a pattern geometry to be foundation paper pieceable, it must be possible to partition its edges into a non-overlapping set of maximal seams (Lemma 4.16).

\subsection{The Sewing Order (a.k.a. Plans)}

The numbers printed on a paper-piecing pattern specify the order in which pieces of fabric for each face of the design should be sewn

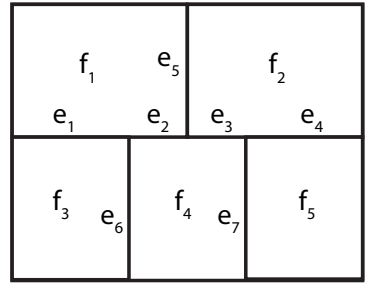

(A) Seams
(B) T-junction

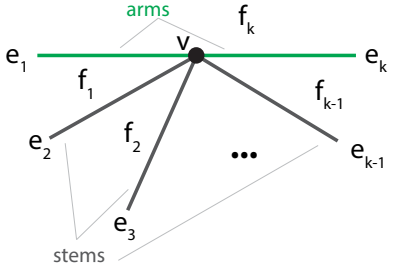

Fig. 4. (A) Simple seams consist of a single edge (e.g., $\left.\left(e_{1}\right),\left(e_{3}\right),\left(e_{5}\right)\right)$. Seams $\left(e_{1}, e_{2}\right)$ and $\left(e_{2}, e_{3}\right)$ are two examples of complex seams because they each share a common face, $\mathrm{f} 1$ and $\mathrm{f} 4$, respectively. However $\left(e_{1}, e_{2}, e_{3}\right)$ is not a seam because those edges do not share a single common face. Note that this design is not foundation paper pieceable by Corollary 4.17 because it contains overlapping maximal seams $\left(e_{1}, e_{2}\right)$ and $\left(e_{2}, e_{3}\right)$. (B) In general, a T-junction is an internal vertex $v$ whose immediate neighborhood consists of two arms (edges, labeled $e_{1}$ and $e_{k}$ ) that are colinear and adjacent in the cyclic ordering, and one or more stems (edges $e_{2}$ through $e_{k-1}$ ) that lie opposite a common face $f_{k}$.

into place. These numbers are a concise way of specifying the full paper-piecing construction process (Section 3.1). We call this full process the (sewing) plan, and in this section we show how to represent it abstractly as a sequence of (sewing) operations. Each operation successively transforms the (sewing) state from an initial state in which nothing has yet been sewn to a final state in which all of the fabric pieces have been sewn along all of the (internal) edges. The definition of foundation paper pieceability is then simply reachability in this state transition system.

Definition 4.6 (Sewing State). A sewing state $\sigma=(\psi, \epsilon)$ of a design $G=(V, F, x)$ with internal edges $E$ consists of (1) $\psi \subseteq F$ the set of faces which have been attached to the paper, and (2) $\epsilon \subseteq E$ the set of edges which have been sewn through.

The initial (or empty) sewing state $\sigma_{0}=(\emptyset, \emptyset)$ of a design $G$ has no fabric attached and no edges sewn. The final (or complete) state $\sigma_{*}=(F, E)$ of a design $G$ has all of the pieces of fabric attached and all of the internal edges sewn.

As discussed (Section 3.2), foundation paper piecing involves two types of sewing operations, sewing the initial seam in which two pieces of fabric are attached along a common edge and sewing subsequent seams in which a new piece of fabric is attached to one or more already-attached pieces along a single seam. We abstract these two sewing operations as transitions from one sewing state that meets specific preconditions to a new sewing state.

Definition 4.7 (Sewing Operations of Type-0 and Type-1). Let $G$ be a design. A sewing operation $O p(s): \sigma \rightarrow \sigma^{\prime}$ is applied at a seam $s$ to transition from sewing state $\sigma$ to sewing state $\sigma^{\prime}$.

A Type- 0 operation $O p^{0}(s): \sigma_{0} \rightarrow \sigma^{\prime}$ requires that $s=(e)$ be a simple seam, with adjacent faces $f_{1}$ and $f_{2}$. It can only be applied to the initial (empty) state, and results in the state $\sigma^{\prime}=\left(\left\{f_{1}, f_{2}\right\},\{e\}\right)$. A Type-1 operation $O p^{1}(s): \sigma \rightarrow \sigma^{\prime}$ is applied at a seam $s=$ $\left(e_{1}, e_{2}, \ldots\right)$ (which may be simple or complex), with common face $f_{0}$ and faces $f_{1}, f_{2}, \ldots$ opposite $f_{0}$. It can only be applied to a state $\sigma=(\psi, \epsilon)$ in which (1) none of the edges of $s$ are already sewn $\left(e_{i} \notin \epsilon\right),(2)$ all of the opposite faces are attached $\left(f_{i} \in \psi\right.$ for $\left.i \geq 1\right)$ 


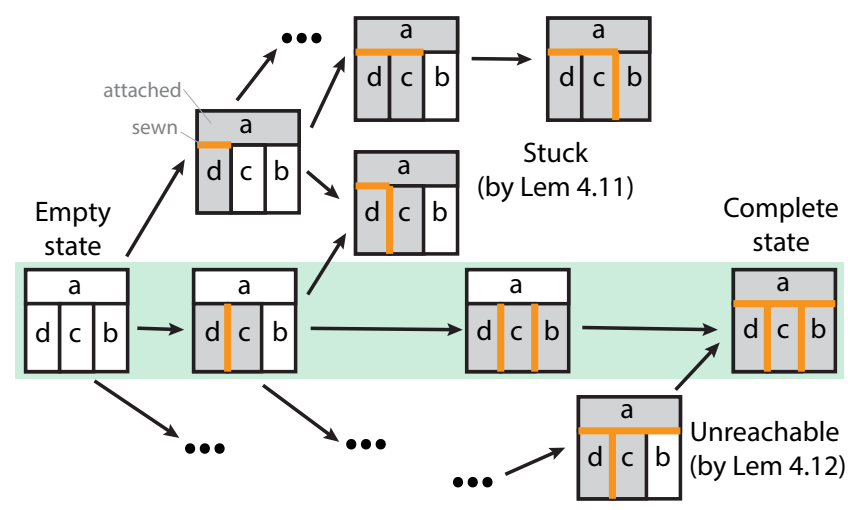

Fig. 5. Every design gives rise to a space of different sewing states (copies of the design above) in which faces may be attached or not and edges sewn or not. States are transitioned between (edges above) via sewing operations. It is impossible to get to the complete state from "stuck" states-in which there is an unsewn edge between attached faces. It is also impossible to get from the initial, empty state to "Unreachable" states-in which there are unattached faces adjacent to sewn edges. We define paper pieceability as the existence of a plan/path to get from the empty state to the complete state in this state-space for a given design.

and (3) the common face is unattached $\left(f_{0} \notin \psi\right)$. After performing the operation, the state is updated to $\sigma^{\prime}=\left(\psi \cup\left\{f_{0}\right\}, \epsilon \cup\left\{e_{1}, e_{2}, \ldots\right\}\right)$.

Remark 4.8. Note that by these definitions, each individual edge may be sewn at most once.

Definition 4.9 (Sewing Plan). A sewing plan for a design $G$ is a sequence of states $\sigma_{0} \rightarrow \sigma_{1} \rightarrow \ldots \rightarrow \sigma_{k}$ from the initial state $\sigma_{0}$ to the some state $\sigma_{k}$ such that exactly one sewing operation (either $O p_{0}$ or $O p_{1}$ ) is applied to transition between each sequential pair of states $\sigma_{i}$ and $\sigma_{i+1}$. A plan is complete when it ends with the complete (final) state $\sigma_{k}=\sigma^{*}$.

With this machinery in place, we can define foundation paper pieceability of a design in a single section, as reachability in our state transition system (Figure 5).

Definition 4.10 (Paper Pieceability of a Design). We say that a design $G$ is paper pieceable iff. there exists a complete sewing plan $\sigma_{0} \rightarrow \cdots \rightarrow \sigma_{k}=\sigma_{*}$ for the design.

\subsection{Properties of Foundation Paper Pieceable Patterns}

We use our definition of foundation paper pieceability to prove that pieceable patterns must always exhibit certain expected properties (e.g., they only contain T-junctions). These properties do not conversely guarantee paper pieceability, but they justify some of the criteria by which designers already guide their design process.

First, we consider recognizable obstructions to completing plans and necessary properties of reachable states. If a plan ever attaches fabric along both sides of an edge without sewing that edge, then the plan can never be completed; neither Type- 0 or Type- 1 operations can sew the edge because both require at least one of the adjacent faces to be unattached.

Lemma 4.11 (Edges That Cannot Be Sewn). Let $G$ be a design and $\sigma=(\psi, \epsilon)$ a sewing state for $G$. If there exists some unsewn edge $e$ between two attached faces $f_{1}$ and $f_{2},\left(e \notin \epsilon\right.$ and $\left.f_{1}, f_{2} \in \psi\right)$, then there does not exist any sequence of sewing operations that will transition $\sigma$ to the complete state $\sigma^{*}$.

\section{See Appendix A for proof.}

Lemma 4.12 (Reachable States). Let $G$ be a design and $\sigma=(\psi, \epsilon)$ a sewing state for $G$, arrived at by applying a sequence of sewing operations to the initial, empty state. Then the following must be true of $\sigma$. (1) If some edge e is sewn (e $\in \epsilon$ ) then both of the faces $f_{1}, f_{2}$ adjacent to e are attached $\left(f_{1}, f_{2} \in \psi\right)$; (2) if some face $f$ is not attached $(f \notin \psi)$ then none of the edges $e$ adjacent to $f$ are sewn $(e \notin \epsilon)$.

\section{See Appendix A for proof.}

As noted in Section 3.2, one well-known rule-of-thumb for foundation paper pieceable patterns is that they can only contain $\mathrm{T}$ junctions (equivalently "I"s or "H"s, see Figure 4B). But "X", "Y". or "L" junctions are not allowed. We formally prove this property.

Definition 4.13 (T-Junction). Let $v$ be an internal vertex in the design $G$. Then we say that $v$ is a $T$-junction when the following statements all hold: (1) $v$ has degree 3 or greater (3 or more edges incident to $v$ ); (2) the edges around $v$ can be cyclically ordered $e_{1}, \ldots e_{k}$ s.t. $e_{1}$ and $e_{k}$ (which are cyclically subsequent) are colinear. (Note that condition 2 is equivalent to saying that there is exactly 180 degrees between some two successive edges around $v$ ) We call $e_{1}$ and $e_{k}$ the arms of the T and all other edges $e_{i}(1<i<k)$ the stems of the $\mathrm{T}$.

Lemma 4.14 (Pieceable Implies T-Junctions). Let $G$ be a paperpieceable design. Then if $v$ is an internal vertex of $G$, it is a T-junction.

\section{See Appendix A for full proof.}

The essence of this proof is a counting argument in the neighborhood of the internal vertex. If the vertex is not a T-junction, then each of the $k$ adjacent edges must be sewn separately in $k$ operations. But at least 2 of the $k$ adjacent faces must be attached after that first operation, and at least one more of the $k$ faces must be attached by each subsequent operation. So we run out of faces-unless by being a T-junction, we are able to sew the two arms of the T-junction in a single operation.

Lemma 4.15 (Complex Seam Implies T-Junctions). Let $G$ be a design (paper pieceable or not), and s a complex seam (i.e., of two or more edges). Then, any internal vertex $v$ of seam $s$ is a T-junction and the arms of the T are edges of the seam.

See Appendix A for proof.

As we saw earlier (Section 4.1), an edge in a pattern geometry can belong to multiple seams. An open question is whether this flexibility matters or not. We will now prove that it does not: that we must sew precisely the set of maximal seams in any complete sewing plan.

Lemma 4.16 (Partition by Maximal Seams). If a design is foundation paper pieceable, then any plan by which it is pieceable (1) partitions the internal edges $E$ into a set of seams $\left(S=\left\{s_{i}\right\}\right.$ which are disjoint, i.e., $s_{i} \cap s_{j}=\emptyset$ for $i \neq j$, and cover the edges, i.e., $E=\bigcup_{i} s_{i}$ ) based on which edges are sewn in the same operation and (2) the set of seams in this partition is exactly the set of maximal seams in the design. 
See Appendix A for full proof.

The proof is similar to our previous counting argument. This time however, the contradiction is created by observing that if the sewn seams do not include all of the maximal seams, then there is some maximal seam that joins together two other colinear seams at a vertex where the two arms of a T-junction are not sewn at the same time. And with that, the previous counting argument follows.

Corollary 4.17 (Overlapping Maximal Seams). If two different maximal seams in a design $G$ are overlapping (i.e., have edges in common) then the design $G$ is not paper pieceable.

Proof. By Lemma 4.16 the maximal seams of a paper pieceable design partition the set of edges, and therefore cannot overlap.

\subsection{Hypergraphs}

In order to state our main theorem characterizing which geometric designs are foundation paper pieceable, we cannot simply rely on acyclicity of graphs as usually understood. For instance, we might conjecture that a design is pieceable if and only if its dual-graph is acyclic. But that is not true, since the dual-graph in the neighborhood of a T-junction is always cyclic, even if the design is pieceable. The problem is that the sewing process takes place on the (maximal) seams of a design, and not on its edges. Therefore we must represent the $k$-way relationships between faces implied by seams, rather than the strictly binary relationships implied by edges. Our solution to these problems is to adapt the concept of hypergraph acyclicity (as studied in databases [Brault-Baron 2016; Fagin 1983]) to the particularities of our setting.

Definition 4.18 (Hypergraph). A hypergraph $H=(N, R)$ is a finite set of nodes $N=\left\{n_{1}, n_{2}, \ldots\right\}$ and finite set of hyperedges $R=\left\{r_{1}, r_{2}, \ldots\right\}$ where each hyperedge is a non-empty subset of the nodes $r_{i} \subseteq N$. We will additionally require that the size or -arity of each hyperedge is at least 2; (Hyperedges of 2-arity are called binary, of 3-arity ternary, etc.) and we will require that the set of hyperedges covers all of the nodes: $\bigcup_{r \in H} r=N$.

Every design gives rise to a "dual" hypergraph as follows.

Definition 4.19 (Dual Hypergraph of a Design). Let $G$ be a design with faces $F$ and maximal seams $S$. Then the dual hypergraph $H_{G}=$ $(N, R)$ of $G$ is defined as follows: (1) the set of nodes $N=F$; (2) for every seam $s \in S$, construct a corresponding hyperedge $r(s)=$ $\left\{f_{1}, f_{2}, \ldots\right\}$ from the set of faces adjacent to the seam $s ; R=\{r(s) \mid s \in$ $S\}$.

Remark 4.20. The binary hyperedges of $H$ correspond to simple seams, which always have exactly two adjacent faces, and the ternary and greater hyperedges of $H$ correspond to complex seams, which always have 3 or more adjacent faces (Figure 6).

One way to characterize acyclicity in a hypergraph is based on a process of reduction [Brault-Baron 2016]. For instance, in the familiar setting of graphs (not a hypergraph), connected acyclic graphs are simply trees and repeatedly plucking the leaves off of the tree will eventually reduce it down to a single node. Conversely, if we can successfully reduce a graph in this manner, it implies the graph is acyclic. Following this notion of acyclicity, we define leaves and their removal/plucking from our dual hypergraphs.

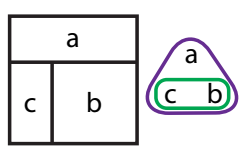

(A) T-junction

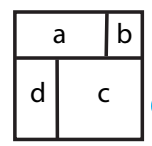

(B) Mondrian

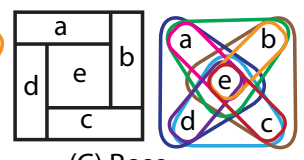

(C) Rose
Fig. 6. Pattern designs and their corresponding dual hypergraphs. Each face is a node in the hypergraph and hyperedges (visualized by colored boundaries) represent seams between two or more faces. Simple seams produce binary hyperedges, while complex seams at T-junctions produce higher order hyperedges. Mondrian (B) and Rose (C) produce cyclic hypergraphs and are not paper pieceable.

Definition 4.21 (Sub-Hypergraph). Let $H=(N, R)$ be a hypergraph. We say that $H^{\prime}=\left(N^{\prime}, R^{\prime}\right)$ is a sub-hypergraph of $H$ when $N^{\prime} \subseteq N$ and $R^{\prime} \subseteq R$, and $H^{\prime}$ is a hypergraph (i.e., all of the nodes are covered by some hyperedge). Given a subset of nodes $N^{\prime} \subseteq N$, we say that $H^{\prime}=\left(N^{\prime}, R^{\prime}\right)$, where $R^{\prime}=\left\{r \mid r \subseteq N^{\prime}\right\}$ is the subhypergraph induced by $N^{\prime}$ provided $H^{\prime}$ is a well-defined hypergraph (i.e., $R^{\prime}$ covers $N^{\prime}$ ).

Definition 4.22 (Leaf of a Hypergraph). Let $H=(N, R)$ be a hypergraph. We say that $r^{\prime} \in R$ is a leaf-edge of $H$ iff. there is some node $n \in r^{\prime}$ and only in $r^{\prime}\left(\forall r \neq r^{\prime}, n \notin r\right)$. We sometimes refer to these nodes as leaf-nodes

Definition 4.23 (Plucking a Leaf). Let $H=(N, R)$ be a hypergraph, and $r$ be a leaf of $H$. Let $L=\left\{n_{1}, n_{2}, \ldots, n_{k}\right\} \subseteq r$ be those nodes of $r$ covered only by $r$ (i.e., $\forall n \in L \forall r^{\prime} \neq r, n \notin r^{\prime}$ ). Then, we say that the sub-hypergraph $H^{\prime}=(N-L, R-\{r\})$ is the result of plucking the leaf $r$ from $H$.

Definition 4.24 (Acyclic Hypergraphs). We define hypergraph acyclicity inductively. The empty hypergraph $H=(\emptyset, \emptyset)$ is acyclic. If $H^{\prime}$ is the sub-hypergraph resulting from plucking a leaf hyperedge $r$ from $H$, and $H^{\prime}$ is acyclic, then $H$ is acyclic.

Remark 4.25. If $H=(N, R)$ consists solely of binary hyperedges, then this definition coincides with the usual definition of graphacyclicity.

The following lemma is simple, but essential, for constructing efficient acyclicity checking algorithms. In effect, it says that the order in which leaves are plucked doesn't matter. So there is no difference between greedily plucking leaves, and exhaustively trying all leaf plucking orders.

Lemma 4.26 (Greedy Leaf Plucking). Let $r^{*}$ be a leaf of a hypergraph $H=(N, R)$, and let $H^{\prime}=\left(N^{\prime}, R^{\prime}\right)$ be any sub-hypergraph of $H$ such that $r^{*}$ is a hyperedge of $H^{\prime}$ as well. Then $r^{*}$ is a leaf of $H^{\prime}$.

$$
\text { See Appendix A for proof. }
$$

\subsection{Main Theorem}

Theorem 4.27. Let $G$ be a design and $H$ its associated hypergraph. Then $G$ is foundation paper pieceable iff. $H$ is acyclic.

See Appendix A for full proof.

The intuition for this theorem is that we can make a correspondence between the sewing process (i.e., sewing plans) and 

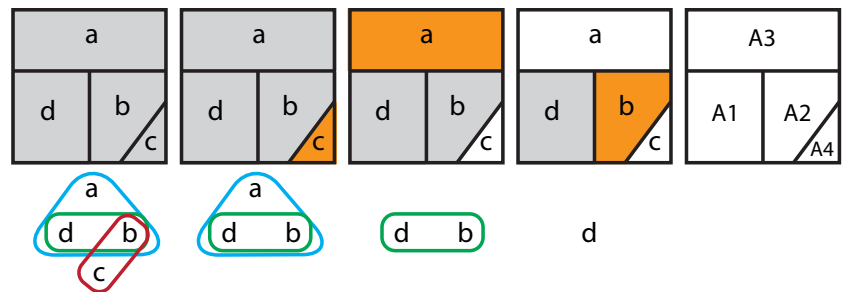

1. Start

2. Pluck c

4. Pluck $b \quad$ 5. Final order
Fig. 7. To check if a pattern design is foundation paper pieceable, we build its dual hypergraph (1) and recursively pluck leaf hyperedges (e.g., hyperedges that contain a node that appears in no other hyperedge) keeping track of the corresponding leaf nodes. Here we first pluck the red hyperedge with leaf node c (2) and then the blue hyperedge with leaf node a (3). With one leaf hyperedge remaining (in green) we randomly choose to pluck leaf node b (4). Reversing the order of the plucked nodes (d, b, a, c) gives us the sewing order for pattern, and we number the faces accordingly (5).

the acyclicity-checking process of plucking leaves from the dual hypergraph. Specifically, the leaf-plucking process aligns with the sewing process in reverse. The first leaf plucked corresponds to the last seam sewn. The proof of the main theorem then is simply a matter of inductively arguing that this correspondence exists and verifying that there is no way that the correspondence can break down.

Key to the proof are two lemmas, which we state here to give a flavor of the argument. The first lemma confirms the intuition that executing a prefix of any complete plan of a design implicitly defines a sub-design. The second confirms that even though leaf hyperedges can have more than one corresponding leaf node, this situation never arises for dual hypergraphs of designs, except for the trivial case. Thus, this second lemma lets us identify the unique face sewn by a sewing operation corresponding to each leaf that we pluck off.

Lemma 4.28 (Prefix Designs Are Well-Defined). Let $G=(V, F, x)$ be a paper pieceable design with at least 3 faces and complete plan $\sigma_{0} \rightarrow \cdots \rightarrow \sigma_{k-1} \rightarrow \sigma_{k}$. Furthermore, let $O p^{1}(s): \sigma_{k-1} \rightarrow \sigma_{k}$ be the final operation of the plan and $\sigma_{k-1}=(\psi, \epsilon)$ s.t. $F=\psi \cup\{f\}$ and $f \notin \psi$. Then (1) $G^{\prime}$, the sub-design induced by $\psi$ is a well-defined sub-design, and (2) $\sigma_{0} \rightarrow \cdots \rightarrow \sigma_{k-1}$ is a complete plan for $G^{\prime}$.

\section{See Appendix A for proof.}

Lemma 4.29 (Leaf Edges Define Unique Leaf Nodes). Let $G$ be a design with 3 or more faces and $H_{G}$ its dual hypergraph. If $r(s)$ is a leaf of $H_{G}$, then there exists a unique node $n^{*} \in r(s)$ that does not occur in any other hyperedge of $H_{G}$. Furthermore, when the seam $s$ is complex, $n^{*}$ is the common face lying along one side of that seam.

See Appendix A for proof.

\section{ALGORITHMIC IMPLEMENTATION}

We apply Theorem 4.27 to check that an input geometric design is foundation paper pieceable by checking that its dual hypergraph is acyclic. If the geometry is paper pieceable, our algorithm can automatically generate all possible sewing orders for it. If it is not paper pieceable, our algorithm highlights the seams that are part of a cyclic substructure so that quilt designers can focus on redesigning these parts of the pattern. Our algorithm proceeds in 4 steps.

(Step 1) Construct maximal seams. We take a SVG pattern design as input and start by constructing a set of seams for each face. Specifically, we cycle through the edges of each face in counterclockwise order and consider each non-boundary edge $e_{i}$. If $e_{i}$ is the first edge, we create a new seam containing it. If $e_{i}$ is colinear with the previous edge $e_{i-1}$ we add it to the seam containing $e_{i-1}$. Otherwise, we create a new seam containing $e_{i}$. To reduce this set of seams to the maximal seams, we first sort the seams by size (i.e., number of edges they contain) and then iterate through the sorted seam list, removing any seam that is a sub-seam of another seam.

(Step 2) Check for overlapping maximal seams. By Corollary 4.17, if the pattern contains overlapping maximal seams, it is not paper pieceable. We check for overlapping seams by checking whether an edge belongs to more than one maximal seam. If so, we mark the design as non-foundation paper pieceable.

(Step 3) Construct dual hypergraph. We construct the dual hypergraph $G$, by treating each face as a node and building a hyperedge for each maximal seam connecting all faces that are adjacent to any edge in the seam. Simple maximal seams produce hyperedges that connect two face nodes, while complex maximal seams produce complex hyperedges connecting three or more face nodes.

(Step 4) Check for acyclicity. Definition 4.24 gives us a procedure for checking the acyclicity of the hypergraph $G$ by sequentially plucking off its leaf hyperedges. Starting from $G$, we first identify all of the leaf hyperedges based on Definition 4.22; specifically, we mark a hyperedge as a leaf if one of its nodes does not belong to any other hyperedge. If the set of leaf hyperedges is empty, $G$ is cyclic, and we mark the design as non-foundation paper pieceable. Otherwise, we pluck off one of the leaf hyperedges - i.e., we remove the hyperedge and its unique leaf node $n_{0}$ (Lemma 4.29) that does not belong to any other hyperedge - to form a new sub-hypergraph $G^{\prime}$. Note that each such plucking removes exactly one node (by Lemma 4.29) and is equivalent to separating one of the faces from the design by cutting along a single seam. We repeat this procedure on $G^{\prime}$ recursively, plucking one leaf hyperedge and corresponding leaf node $n_{i}$ at each recursive step. If we successfully reduce $G$ to a hypergraph with one binary hyperedge on two nodes, then we've succeeded in demonstrating acyclicity. By reversing the sequence of plucked nodes $\left(n_{k}, n_{k-1}, \ldots, n_{0}\right)$ we obtain a valid sewing order for attaching the faces (Figure 7). Otherwise, our recursive procedure ends with a non-empty sub-hypergraph $G^{\prime}$ that contains no leaves and is therefore cyclic. We mark the corresponding design as nonfoundation paper pieceable, and our interface highlights in red all of the maximal seams in the final sub-hypergraph $G^{\prime}$ forming the cycle (Figures 10 and 11).

Greedily choosing leaves to pluck has no bearing on whether the process succeeds, thanks to Lemma 4.26. However, we can produce all valid sewing orders using the following approach. At each iteration of our leaf-plucking procedure, we maintain a list of leaf hyperedges and instead of arbitrarily choosing one leaf to pluck off, we systematically pluck off each leaf in the list and consider 
(A) Basket 1

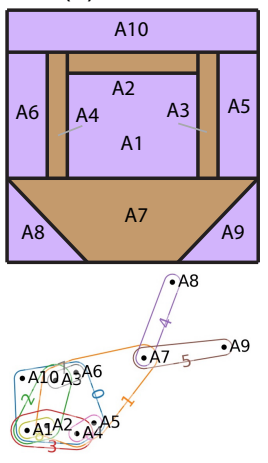

Sections:

Sections:

10

Complex seams:

Sewing orders: 96
(B) Basket 2
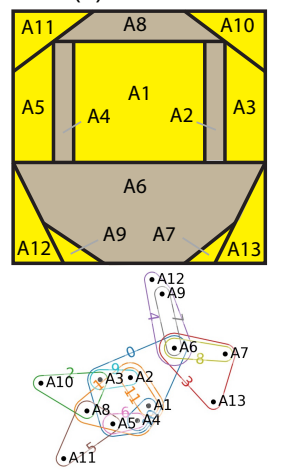

Sections:

Faces:

Faces: 13

Simple seams:

Complex seams:

Sewing orders: 15838
(C) Twin flowers

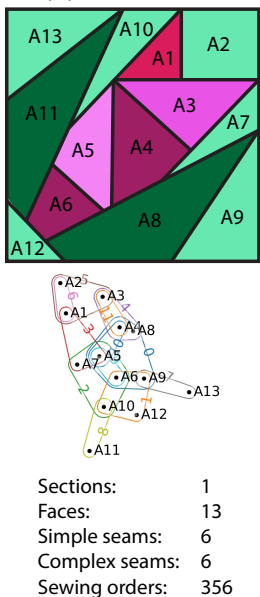

(D) Lily
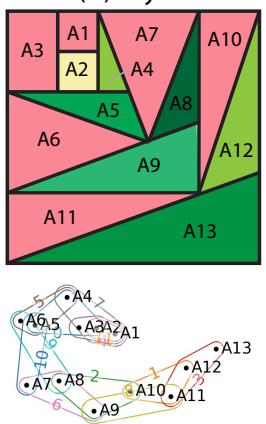

$\begin{array}{ll}\text { Sections: } & 1 \\ \text { Faces: } & 13\end{array}$

Simple seams: 4

Complex seams: 6

Sewing orders: 24
(E) Hot air balloon
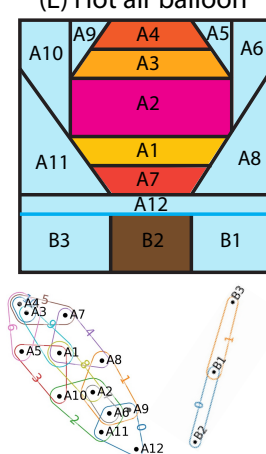

Sections: $\quad 2$

Faces: $\quad 12,3$

Simple seams: $\quad 4,3$

Complex seams: 3,0

Sewing orders: 1972,4
(F) Mailbox

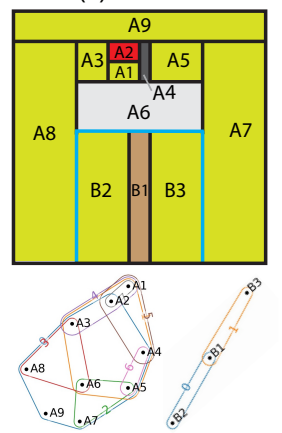

Sections: 2

Faces: $\quad 9,3$

Simple seams: $\quad 4,3$

Complex seams: 3,0

Sewing orders: 12,4

Fig. 8. Six patterns from Doak's book [2011] with their dual hypergraphs. Even for relatively simple designs, the hypergraphs can be quite complex. Designs with many viable sewing orders (e.g., Basket 2) often have multiple leaf hyperedges available to pluck at any given step of our algorithm. (See if you can identify the leaf hyperedges yourself.) For the Hot Air Balloon and Mailbox patterns, Doak suggests using traditional, non-paper piecing techniques to sew a few of the faces into the design. We specify section boundaries for these two designs and let our algorithm find paper pieceable sewing orders for each resulting section.

all possible sub-hypergraphs in the next iteration. This procedure necessarily enumerates all valid permutations of the hyperedges, which correspond to the sewn seams (Lemma 4.16) and are in correspondence with the faces to be numbered (Lemma 4.29).

\section{RESULTS}

We have applied our algorithm to a variety of input designs to automatically generate foundation paper pieceable sewing orders (Figure 8) and also incorporated our algorithm into an interactive quilt design tool (Figure 9-11), which we have informally evaluated with a few quilters (Figures 12-13).

\subsection{Computing viable sewing orders}

To validate our algorithm we converted all 50 patterns in Doak's [2011] "50 Little Paper-Pieced Blocks: Full-Size Patterns to Mix \& Match" into SVGs, making sure to mark section boundaries in the multisection designs. Table 1 shows that these patterns cover a large range of design possibilities with varying numbers of sections, faces, and seams (both simple and complex). Figure 8 shows examples of various patterns from the book with their dual hypergraphs and one of the viable sewing orders (indicated by the numbering of the faces) generated by our algorithm. As shown in these examples, the number of viable sewing orders often depends on the number of faces and complex seams contained in pattern design.

For example, although both the "Twin Flowers" and "Lily" designs (Figure 8C,D) have 13 faces, "Twin Flowers" has 356 sewing orders, versus 24 for "Lily". The nested T-junctions form many complex seams in the "Lily" that enforce stricter ordering constraints on the faces. T-junctions and their seams reduce the number of faces associated with leaf hyperedges because the faces around the stem(s) of the T-junction are always part of at least two hyperedges (Figure 6A). The hypergraphs for designs that yield more sewing orders tend to
Table 1. We have generated all sewing orders for the 50 patterns in a wellknown book on paper piecing [Doak 2011]. We report the range of the number of faces and seams per section. Our algorithm computes the number of sewing orders for each section of each design.

\begin{tabular}{rrr|rr|r}
$\begin{array}{r}\text { Num. } \\
\text { Patterns }\end{array}$ & $\begin{array}{r}\text { Num. } \\
\text { Sections }\end{array}$ & $\begin{array}{c}\text { Num. } \\
\text { Faces }\end{array}$ & $\begin{array}{c}\text { Num. Seams } \\
\text { Simple }\end{array}$ & $\begin{array}{r}\text { Num. } \\
\text { Complex }\end{array}$ & Sew Orders \\
\hline 35 & 1 & 2 to 18 & 2 to 10 & 2 to 12 & 2 to 15838 \\
12 & 2 & 3 to 12 & 1 to 8 & 0 to 4 & 4 to 1972 \\
1 & 3 & 5 to 12 & 3 to 4 & 0 to 7 & 16 to 644 \\
2 & 4 & 4 to 9 & 2 to 4 & 1 to 2 & 2 to 24
\end{tabular}

start with more leaf hyperedges (equivalently leaf nodes included in a single hyperedge), that can be plucked off in any order. While "Basket 1" and "Basket 2" are similar in appearance (Figure 8A,B), "Basket 2" starts with 4 leaf hyperedges - the hyperedges containing A10, A11, A12, and A13. "Basket 1" only starts with 3 such leaf hyperedges containing A8, A9, and A10.

We note that for 5 of Doak's patterns designs, the author does not provide a sewing order for a subset of the pieces in the design. Instead Doak instructs quilters to "cheat" and sew these pieces using traditional quilting techniques rather than foundation paper piecing. With our algorithm we can specify a section boundary, and it is able to identify viable sewing orders for each section. This lets quilters make use of the stability and precision of paper piecing for each section and only requires using traditional sewing to join the sections together (Figure 8E,F)

\subsection{Interactive design tool}

We have also developed an interactive tool for designing quilt patterns that uses our leaf-plucking algorithm to provide real-time feedback on the paper pieceability of the design as users draw seams. In Figures 9-11 we walk through how we designed patterns for a cactus, a camera, and a house using the tool. In all three cases the tool lets us focus on the visual design of the pattern and indicates a paper 

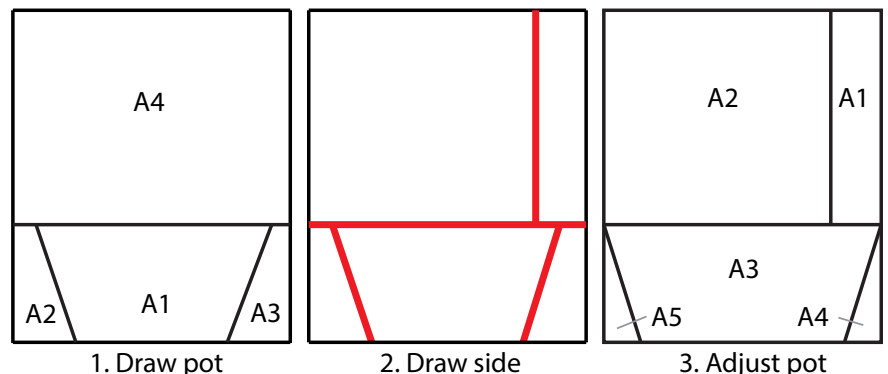

3. Adjust pot

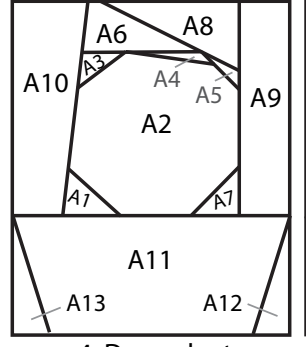

4. Draw plant

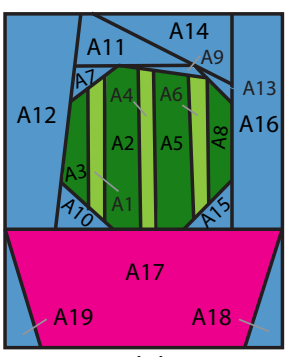

5. Final design

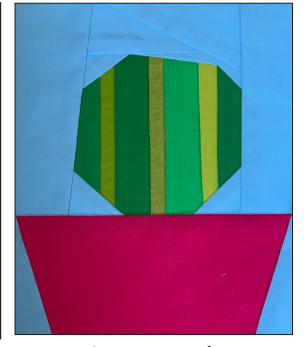

Sewn result

Fig. 9. In designing this barrel cactus pattern, adding a vertical seam at the top right (step 2) creates a problem where we have multiple T-junctions with stems on either side of the horizontal edge causing overlapping maximal seams (similar to the problematic configuration in Figure 4A). The red seams alert us to the problem, and we adjust the seams on the side of the pot, moving their top points to the boundary of the pattern (step 3). This removes the problem, without drastically altering the appearance of the design and without introducing a section boundary. The pattern is again single section paper pieceable, as indicated by the sewing order numbering, and remains so as we add additional details to the cactus (steps 4-5).

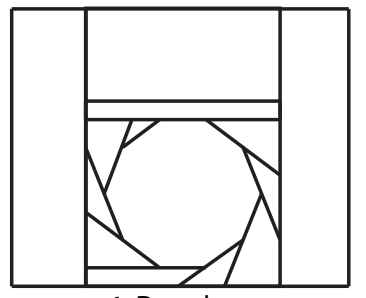

1. Draw lens

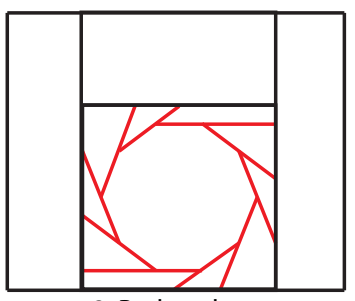

2. Redraw lens

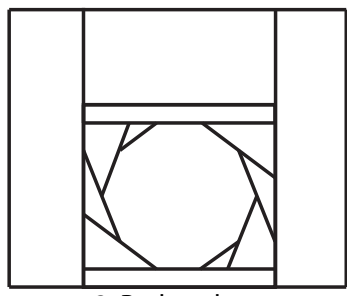

3. Redraw lens

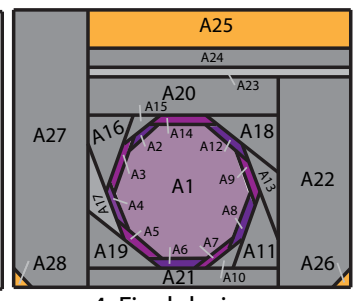

4. Final design

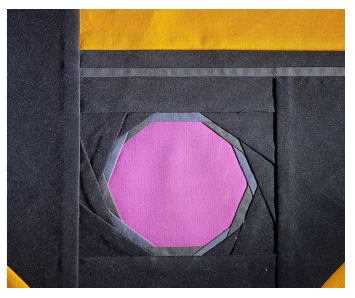

Sewn result

Fig. 10. We start the design of this camera pattern by focusing on the lens area. While our first design (step 1) is paper pieceable, we would like it to be more symmetric. So we erase the left side of the horizontal edge at the top of the lens and extend two of the angled edges (step 2). But our tool indicates that this relatively small change creates a large cycle including all of the seams in the lens by turning them red (this is similar to the problematic Rose design of Figure 2D). To resolve the problem, we undo the change and add horizontal bars at the top and bottom of the lens to make it both symmetric and paper pieceable (step 3). We then continue to add detail to the design while maintaining a single section paper pieceable pattern (step 4). Our tool helps us catch and resolve the design problem before we get too far ahead in our design.

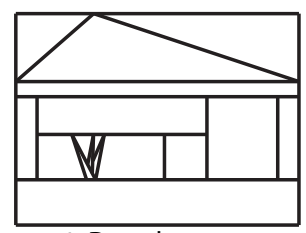

1. Draw house

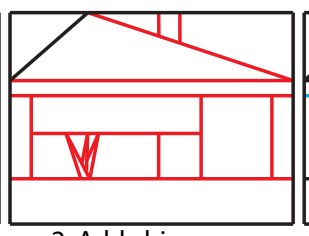

2. Add chimney

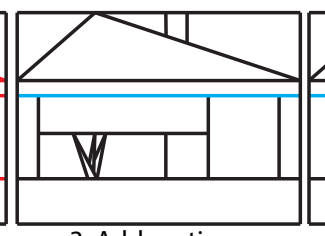

3. Add section

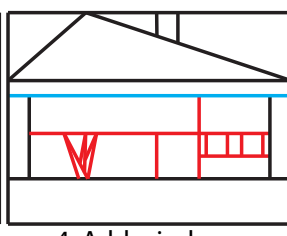

4. Add windows

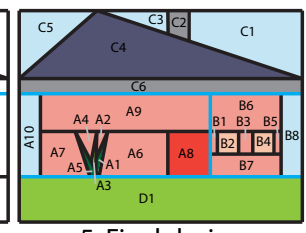

5. Final design

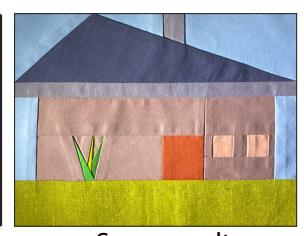

Sewn result

Fig. 11. For this modern house pattern we start with a basic design (step 1) and then add a chimney (step 2), but the tool shows us that the design is no longer paper pieceable and that many seams are involved in the cycle. Noticing this problem without our tool would be challenging because the problematic T-junctions are separated by two faces, which are part of the roof (similar to the Separated Mondrian block of Figure 2C). We fix the problem by adding a long horizontal section boundary line just below the roof (blue) that splits the pattern into two paper pieceable sections (step 3). We then add windows (step 4), which again creates a problem in the lower section because of overlapping maximal seams where the door and window seams meet. We add two more section lines to make the block paper pieceable. Although we could have made this design with just three sections (e.g., by making the window panel its own section), we choose to make 4 sections to make joining the sections easier with long, straight seams.

pieceable sewing order by numbering the polygons whenever the design is foundation paper pieceable. As shown in the supplemental video, our interface chooses one of the viable sewing orders as a default but also allows users to scroll through the other viable orderings and select an alternate order if they would prefer a different one.

When the design is not paper pieceable, the interface alerts us by highlighting the seams that are part of the hypergraph cycle in red. We can then adjust the geometry (e.g., cactus, camera) or divide the design into sections (e.g., house) to resolve the problem. While the interface does not require us to immediately resolve such paper pieceability problems, by indicating that the problem exists, it provides a cue that we should consider resolving the problem before going significantly further with the design.

\subsection{Informal user study}

We asked three quilters with varying levels of experience in foundation paper piecing (P1 had no paper piecing experience, $\mathrm{P} 2 \mathrm{had}$ made a few paper pieced quilts, and P3 has been selling foundation paper piecing patterns for 30 years) to use our tool to design patterns. Each participant first drew a quilt pattern from an image 


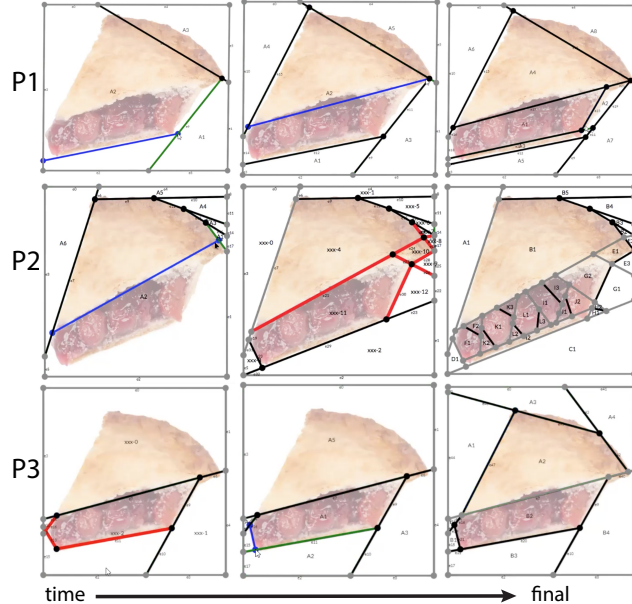

(A) Pie slice

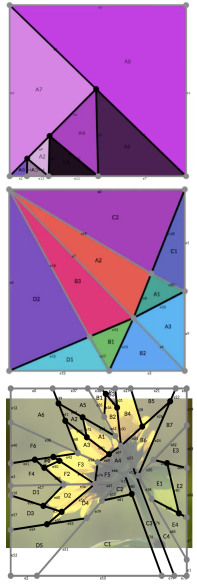

(B) Participant's choice
Fig. 12. Our study participants (P1, P2, \& P3) each drew different designs based on the same image of a pie slice $(A)$ and then designed original patterns by free-hand drawing in the tool (P1 \& P2) or tracing an image (P3) (B). P1's pie slice design had 1 section and 8 faces, P2's had 12 sections and 27 faces, and P3's had 2 sections and 9 faces. For the participant's choice designs, P1's had 1 section and 8 faces, P2's had 4 sections and 10 faces, and P3's had 6 sections and 32 faces.

of a slice of cherry pie (Figure 12A). Then, we asked them to draw 1 to 3 additional pattern designs and left them free to work from their own previous sketches or an underlying photograph of their choice or to draw-freehand in our tool (Figure 12B). We asked them to think-aloud and describe what they were doing, as they created their designs.

All participants successfully completed the pie slice design and at least one additional pattern of their choice. They noticed whenever the seams were highlighted in red and understood that the pattern was no longer paper pieceable. While they would sometimes continue to draw a few additional seams in the non-paper pieceable condition, they would usually resolve the paper pieceability problem quickly after seeing it. They resolved the problem either by locally redesigning the seams (e.g., adding or erasing seams) or by splitting the pattern into multiple sections.

After completing their designs, we asked the participants for general feedback about our tool and how it might fit within their existing design practices. Feedback on a 5-point Likert scale was generally positive. All three participants found the tool helpful ( $\mu=$ 3.7), easy-to-use ( $\mu=4.3$ ), and less effort than manually determining pattern orders $(\mu=4.3)$. They all noted and appreciated the regular feedback from the tool while they were drawing. P1 and P2 explicitly said it allowed them to fix issues quickly when they occurred, rather than waiting until they had created a detailed design only to find it was not paper pieceable. After designing four patterns using our tool, working from their own sketches and photographs, P2 said, "The ordering part is brilliant. It takes the whole computation out of the way so you can just play with it." P2 chose to continue to work in the tool and sewed a full quilt based on the designs they created (Figure 13).
Participants also suggested several features for improving the interface. For example, P3 noted that an algorithm for picking the default sewing ordering based on adding the largest area faces first would better correspond to the way they typically work. Attaching larger pieces first provides more stability for the smaller pieces added later. We plan to investigate other such criteria for choosing among the viable orders in future work. Others suggested lowerlevel features, such as providing alignment tools and a background grid to facilitate drawing and including a gallery of previous designs for inspiration.

\section{LIMITATIONS AND FUTURE WORK}

We have formalized the foundation paper piecing quilt construction process and developed an algorithm to check if a given geometric design is paper pieceable. Our approach, however, does rely on a couple of underlying assumptions that limit its ability to produce all possible input geometries. While many of these limitations are inherent to foundation paper piecing as we discussed in Section 3, it may also be possible to lift some of them in future work.

\subsection{Curved interior seams}

A central assumption of foundation paper piecing patterns is that all interior seams are composed of straight lines [Sharp 2018]. While the boundary seams may be curved, in general it is very difficult to stitch curved seams because the fabric in the seam allowance either has to bunch together or has to stretch apart, depending on whether the face is concave or convex at the seam. Ensuring the faces on both sides of the seam lie flat, typically requires cutting slits and notches in the seam allowance and manually sewing the seam using traditional techniques without paper. However, recently Kaptein [2020] has demonstrated that it is possible to paper piece shallow curved interior seams using stiff paper, copious amounts of glue and careful stitching to keep the fabric lying flat. Extending our mathematical formalization to handle such curved interior seams, perhaps using some of the techniques used to analyze curved paper folding [Kilian et al. 2008] could enable computational approaches to working with such seams under the constraints of foundation paper piecing.

\subsection{Applique and English paper piecing}

Applique and English paper piecing are quilting techniques that can use paper guides for parts of the quilting process but impose different constraints on the pattern design. For example, in English paper piecing, the fabric is never sewn directly to the paper. Developing a formal theory for these methods and developing a design tool for them could be explored in future work.

\subsection{Automatic sectioning or geometric redesign}

Our interactive design tool alerts users when the pattern is not paper pieceable but leaves it up to users to choose how to address the problem. Automatically suggesting how to split the pattern into multiple sections or how to redesign the seam geometry so that the pattern is paper pieceable could be especially useful for inexperienced designers. The challenge is to identify the variety of criteria designers might use to choose the section boundary or to 


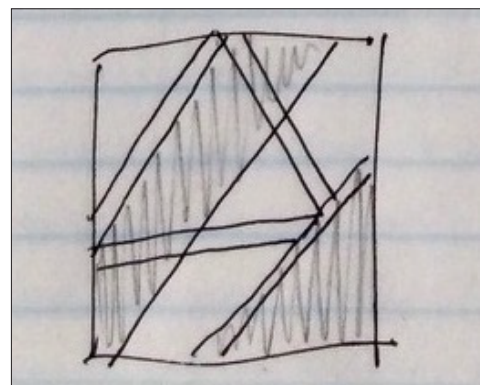

(A) Sketch

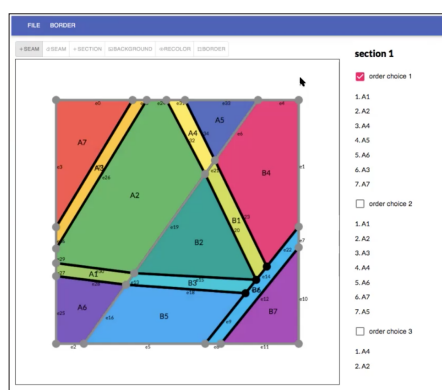

(B) Design in our tool

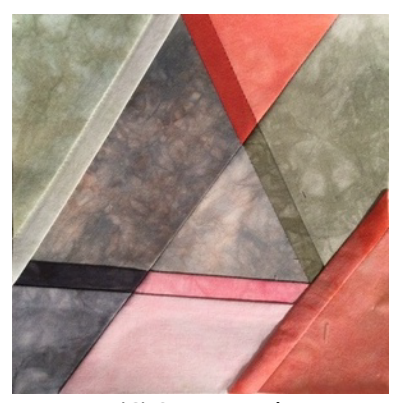

(C) Sewn result

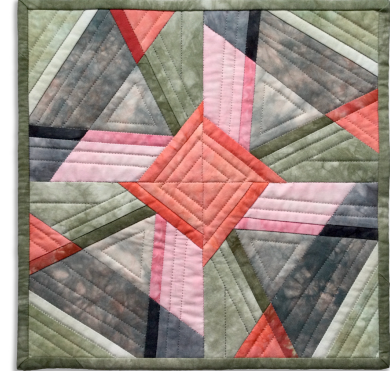

(D) Finished quilt

Fig. 13. P2 started with a sketch of a potential design (A), but could not figure out how to generate a sewing order for it. They used our tool to generate a paper pieceable version of the design containing 2 sections and 14 faces (B). They used the resulting order to sew the design (C) and then composed four of these blocks into a full mini-quilt (D) by layering the quilt top with batting and backing fabric and stitching quilting lines to echo the design of the blocks and hold the layers together. These additional quilting lines give texture to the quilt.

remove and modify the seams. A related direction would be to fully automate the geometric design process so that given an input image, the tool could automatically generate a foundation paper pieceable geometric design for it.

\section{CONCLUSION}

Although the modern process of foundation paper piecing was only introduced in the 1970s [Mahoney 2016], the stability and precision it adds to quiltmaking have made it very popular. But because the process constrains pattern geometry, few quilters design their own foundation paper pieceable patterns. We have shown how to mathematically formalize the paper piecing process and have used this formalization to develop an interactive quilt design tool that automates the tedious step of determining whether a design is paper pieceable. We believe that our formalization and the resulting design tool have the potential to significantly increase the number of quilters who design their own foundation paper pieceable patterns.

\section{ACKNOWLEDGMENTS}

We thank Doug James and Pat Hanrahan for their advice and feedback on this work and the quilters who participated in our study and shared their paper piecing insights and design talent with us.

\section{REFERENCES}

Ingrid Alteneder. 2020. Adorable Animal Quilting. Page Street.

Aric Bartle, Alla Sheffer, Vladimir G. Kim, Danny M. Kaufman, Nicholas Vining and Floraine Berthouzoz. 2016. Physics-Driven Pattern Adjustment for Direct 3D Garment Editing. ACM Trans. Graph. 35, 4, Article 50 (July 2016), 11 pages. https://doi.org/10.1145/2897824.2925896

Floraine Berthouzoz, Akash Garg, Danny M. Kaufman, Eitan Grinspun, and Maneesh Agrawala. 2013. Parsing Sewing Patterns into 3D Garments. ACM Trans. Graph. 32, 4, Article 85 (July 2013), 12 pages. https://doi.org/10.1145/2461912.2461975

Bernd Bickel, Paolo Cignoni, Luigi Malomo, and Nico Pietroni. 2018. State of the Art on Stylized Fabrication. Computer Graphics Forum 37 (2018). http://vcg.isti.cnr.it/ Publications/2018/BCMP18

Johann Brault-Baron. 2016. Hypergraph Acyclicity Revisited. ACM Comput. Surv. 49, 3, Article 54 (Dec. 2016), 26 pages. https://doi.org/10.1145/2983573

Christopher Carlson, Nina Paley, Theodore Gray, et al. 2015. Algorithmic quilting. In Proceedings of Bridges 2015: Mathematics, Music, Art, Architecture, Culture. Tessellations Publishing, 231-238.

Marge M Coahran and Eugene Fiume. 2005. Sketch-Based Design for Bargello Quilts.. In SBM. 165-174.

Arnout Cosman. 2012. Quilt Assistant v2.24. https:/quiltassistant.com/
Erik D Demaine and Martin L Demaine. 2002. Recent results in computational origami. In Origami3: Third International Meeting of Origami Science, Mathematics and Education. 3-16.

Phoebe Moon Designs. 2020. The seven deadly sins of foundation paper piecing. https: //phoebemoon.com/tutorials/paper-piecing.htm

Mario Deuss, Daniele Panozzo, Emily Whiting, Yang Liu, Philippe Block, Olga SorkineHornung, and Mark Pauly. 2014. Assembling self-supporting structures. ACM Trans. Graph. 33, 6 (2014), 214-1.

Carol Doak. 2011. 50 Little Paper-Pieced Blocks: Full-Size Patterns to Mix \& Match. C\&T Publishing.

ElectricQuilt. 2017. Electric Quilt 8 (EQ8). https://electricquilt.com/

Ronald Fagin. 1983. Degrees of Acyclicity for Hypergraphs and Relational Database Schemes. F. ACM 30, 3 (July 1983), 514-550. https://doi.org/10.1145/2402.322390

Yohsuke Furuta, Nobuyuki Umetani, Jun Mitani, Takeo Igarashi, and Yukio Fukui 2010. A Film Balloon Design System Integrated with Shell Element Simulation.. In Eurographics (Short Papers). 33-36.

Akash Garg, Andrew O Sageman-Furnas, Bailin Deng, Yonghao Yue, Eitan Grinspun, Mark Pauly, and Max Wardetzky. 2014. Wire mesh design. ACM Transactions on Graphics 33, 4 (2014).

Hayley Grzych. 2018. Beginner-friendly foundation paper piecing. https://weallsew. com/beginner-friendly-foundation-paper-piecing

Emmanuel Iarussi, Wilmot Li, and Adrien Bousseau. 2015. WrapIt: Computer-Assisted Crafting of Wire Wrapped Jewelry. ACM Trans. Graph. 34, 6, Article 221 (Oct. 2015), 8 pages. https://doi.org/10.1145/2816795.2818118

Takeo Igarashi and John F Hughes. 2002. Clothing manipulation. In Proceedings of the 15th annual ACM symposium on User interface software and technology. 91-100.

Yuki Igarashi and Takeo Igarashi. 2010. Holly: A drawing editor for designing stencils. IEEE Computer Graphics and Applications 30, 4 (2010), 8-14.

Yuki Igarashi and Jun Mitani. 2015. Patchy: An interactive patchwork design system. In ACM SIGGRAPH 2015 Posters. 1-1.

Sylvia Kaptein. 2020. Curved paper piecing: again, but different. https://blog.bernina. com/en/2020/01/curved-paper-piecing-again-but-different/

Lily Kerns. 2020. Designing a Foundation Pieced Block from a Photograph. https://www.academyofquilting.com/library/free-lessons/designing-afoundation-pieced-block-from-a-photograph/

M. Kilian, S. Flöry, Z. Chen, N. J. Mitra, A. Sheffer, and H. Pottmann. 2008. Curved Folding. ACM Transactions on Graphics 27, 3 (2008), \#75, 1-9.

Maria Larsson, Hironori Yoshida, Nobuyuki Umetani, and Takeo Igarashi. 2020. Tsugite: Interactive Design and Fabrication of Wood Joints. In Proceedings of the 33rd Annual ACM Symposium on User Interface Software and Technology. 317-327.

Yifei Li, David E Breen, James McCann, and Jessica Hodgins. 2019. Algorithmic Quilting Pattern Generation for Pieced Quilts. (2019), 9.

Chenxi Liu, Jessica Hodgins, and James McCann. 2017. Whole-cloth quilting patterns from photographs. In Proceedings of the Symposium on Non-Photorealistic Animation and Rendering - NPAR '17. ACM Press, Los Angeles, California, 1-8. https://doi.org/ $10.1145 / 3092919.3092925$

Nancy Mahoney. 2016. Learn to Paper Piece: A visual guide to piecing with precision. Martingale.

James McCann, Lea Albaugh, Vidya Narayanan, April Grow, Wojciech Matusik, Jennifer Mankoff, and Jessica Hodgins. 2016. A compiler for 3D machine knitting. ACM Transactions on Graphics 35, 4 (July 2016), 1-11. https://doi.org/10.1145/2897824. 2925940 
Jun Mitani and Hiromasa Suzuki. 2004. Making papercraft toys from meshes using strip-based approximate unfolding. ACM transactions on graphics (TOG) 23, 3 (2004), 259-263.

Yuki Mori and Takeo Igarashi. 2007. Plushie: an interactive design system for plush toys. In ACM SIGGRAPH 2007 papers. 45-es.

Vidya Narayanan, Lea Albaugh, Jessica Hodgins, Stelian Coros, and James McCann. 2018. Automatic machine knitting of 3D meshes. ACM Transactions on Graphics (TOG) 37, 3 (2018), 1-15

PreQuilt. 2020. PreQuilt. https://prequilt.com/

Quiltster. 2020. Quiltster | Digital Quilt Planner. https://www.quiltster.com/

Sarah Elizabeth Sharp. 2018. Adventures in Paper Piecing \& Design. Stash Books.

Mélina Skouras, Bernhard Thomaszewski, Bernd Bickel, and Markus Gross. 2012. Com putational design of rubber balloons. In Computer Graphics Forum, Vol. 31. Wiley Online Library, 835-844.

Mélina Skouras, Bernhard Thomaszewski, Peter Kaufmann, Akash Garg, Bernd Bickel, Eitan Grinspun, and Markus Gross. 2014. Designing inflatable structures. ACM Transactions on Graphics (TOG) 33, 4 (2014), 1-10.

Gillian Smith. 2017. Generative Design for Textiles: Opportunities and Challenges for Entertainment AI.. In AIIDE. 115-121.

The Quilting Company. 2017. Quilting in America 2017. https://fabshopnet.com/wpcontent/uploads/downloads/qia_summary.pdf

Cesar Torres, Wilmot Li, and Eric Paulos. 2016. ProxyPrint: Supporting Crafting Practice through Physical Computational Proxies. In Proceedings of the 2016 ACM Conference on Designing Interactive Systems (DIS '16). Association for Computing Machinery, New York, NY, USA, 158-169. https://doi.org/10.1145/2901790.2901828

Nobuyuki Umetani, Danny M Kaufman, Takeo Igarashi, and Eitan Grinspun. 2011 Sensitive couture for interactive garment modeling and editing. ACM Trans. Graph 30, 4 (2011), 90

Katja Wolff and Olga Sorkine-Hornung. 2019. Wallpaper Pattern Alignment along Garment Seams. ACM Trans. Graph. 38, 4, Article 62 (July 2019), 12 pages. https: //doi.org/10.1145/3306346.3322991

Linda Worland. 2020. Designing Paper Pieced Patterns. https://www.paperpanache. com/designing-paper-piecing

Jiaxian Yao, Danny M Kaufman, Yotam Gingold, and Maneesh Agrawala. 2017. Interactive design and stability analysis of decorative joinery for furniture. $A C M$ Transactions on Graphics (TOG) 36, 2 (2017), 1-16.

\section{A APPENDIX: DETAILED PROOFS}

Proof of 4.11. Consider the hypothesis $e \notin \epsilon$ and $f_{1}, f_{2} \in \psi$ as an invariant. Type- 0 operations must preserve this invariant because they can only be applied to an edge when both adjacent faces are unattached. Type- 1 operations must preserve this invariant because they can only be applied to a seam containing $e$ when either $f_{1}$ or $f_{2}$ is unattached. Since every reachable state from $\sigma$ must satisfy the invariant, the complete state $\sigma^{*}$ (in which $e$ is sewn) is unreachable.

Proof of 4.12. By induction. Since no edge $e$ is sewn in $\sigma_{0}$, the base case holds. Inductively, a state $\sigma^{\prime}$ is reached by applying a Type- 0 or Type- 1 operation to some prior state $\sigma$ in which the invariant holds. In $\sigma^{\prime}$, if $e$ is a sewn edge, then either $e$ is sewn in $\sigma$, in which case it's adjacent faces are present by the inductive hypothesis, or $e$ was just sewn by the operation. In the latter case, the post-conditions of both type- 0 and type- 1 operations require that the faces on both sides of the edge are attached.

Proof of 4.14. To show (1) suppose $v$ has degree less than 3. Since $G$ is a well-formed planar mesh, $v$ cannot have degree 1 . If $v$ has degree 2 , then the two edges incident to it $e_{1}$ and $e_{2}$ cannot be colinear, or else we would violate condition 2 of well-formedness (Def. 4.1) for planar meshes. Therefore $e_{1}$ and $e_{2}$ cannot be sewn together in a common seam; they must be sewn separately in two different sewing operations. While considering the different possibilities, let $f_{1}$ and $f_{2}$ be the two faces adjacent to the vertex $v$. We will observe what might happen in this local neighborhood under the original hypothesis that $G$ is paper pieceable, and if every possibility yields a contradiction, then we may conclude that $v$ must have degree 3 or greater. Without loss of generality, assume that $e_{1}$ is sewn before $e_{2}$. By Lem.4.12, $f_{1}$ and $f_{2}$ must be attached after $e_{1}$ is sewn, yet $e_{2}$ remains unsewn. Therefore, by Lem.4.11, it is impossible to complete this sewing plan; a contradiction.
To show (2), it is evident that the edges can be cyclically ordered; let $E(v)=\left\{e_{1}, \ldots e_{k}\right\}$ be those cyclically ordered edges around $v$ and $F(v)=$ $\left\{f_{1}, \ldots f_{k}\right\}$ the subsequent cyclically ordered faces. Then to prove (2) by contradiction, we assume that there are no two subsequent edges which are contiguous and colinear. No two of $e_{1}, \ldots, e_{k}$ lie in a common seam; each must be sewn by a different sewing operation. We now make another counting argument. Let $\# \psi(v)$ be the number of attached faces around $v$ and $\# \epsilon(v)$ the number of sewn edges in some state $\sigma=(\psi, \epsilon)$. Note that in the complete state $\# \psi(v)=\# \epsilon(v)$. Let $e^{\prime}$ be the first edge that is sewn, and $\sigma^{\prime}=\left(\psi^{\prime}, \epsilon^{\prime}\right)$ the resulting state. Since $e^{\prime}$ is the first edge sewn, \# $\# \epsilon^{\prime}(v)=1$,

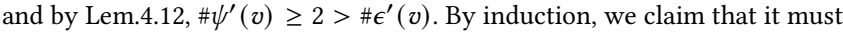
remain true that $\# \psi(v)>\# \epsilon(v)$. To see why, consider the effect of any possible sewing operation on these counts. If that operation does not sew an edge in $E(v)$, then \# $\epsilon(v)$ cannot increase; if that operation is a Type- 0 operation applied to some $e \in E(v)$, then \# $\psi(v)$ increases by 2 while $\epsilon(v)$ only increases by 1 ; finally if that operation is a Type- 1 operation applied to some $e \in E(v)$, then both $\epsilon(v)$ and $\psi(v)$ increase by exactly 1 . Since any state reachable from the initial state has $\# \psi(v)>\# \epsilon(v)$, the complete state is unreachable; a contradiction.

Proof of 4.15. Let $e_{1}, e_{2}$ be the two edges in the sequence $s$ adjacent to $v$. By Def.4.3, $e_{1}$ and $e_{2}$ must be colinear and share a common face $f$. Therefore, all other edges incident to $v$ must lie on the opposite side of $s$ from $f$. Furthermore, by Def.4.1(2), there must be at least one other edge incident to $v$ besides $e_{1}$ and $e_{2}$.

Proof of 4.16. (1) follows immediately from the observation that each edge can be sewn at most once (in one or another sewing operation), and that a complete plan must have sewn all of the internal edges. To see why (2) holds, suppose that the set of sewn seams (which partition the internal edges) is not identically the set of maximal seams. Then there must exist some maximal seam $s^{\prime}$ that was not sewn. Furthermore, $s^{\prime}$ must be complex and overlap with at least two other seams. If not, then either $s^{\prime}$ must include some edge not contained in any other seam (in which case $s^{\prime}$ must have been sewn, violating the hypothesis) or $s^{\prime}$ must be entirely contained within some other seam (violating the hypothesis that $s^{\prime}$ is maximal).

So, $s^{\prime}$ is complex, and overlaps two seams: $s_{1}$ and $s_{2}$. Then $s^{\prime}, s_{1}$ and $s_{2}$ must all be colinear and we may assume that if $s^{\prime}$ overlaps more than two seams, $s_{1}$ and $s_{2}$ are chosen from those s.t. they are contiguous. The vertex $v$ lying between $s_{1}$ and $s_{2}$ lies between edges of $s^{\prime}$, and so we know it must be an internal vertex. By Lemma 4.14, this internal vertex $v$ must be a T-junction, with arms $e_{1}$ and $e_{k}$ that are edges of $s_{1}$ and $s_{2}$ respectively. However, observe that in our plan $s_{1}$ and $s_{2}$ are sewn separately, and therefore that $e_{1}$ and $e_{k}$ are sewn separately. Consequently, we find ourselves back in the same conundrum exhibited in the proof of Lemma 4.14. Whether a Type-0 or Type- 1 operation is the first operation to place down a face adjacent to $v$, we are left with more edges around $v$ to sew than we have faces around $v$ left to attach, and no way to sew multiple such edges per face. Therefore we have reached our ultimately desired contradiction showing that there cannot be any maximal seams that are not already accounted for among the sewn seams of a complete plan.

Proof of 4.26. By the definition of a leaf, there must exist a node $n \in r^{*}$ s.t. $\forall r \in R, r \neq r^{*} \Longrightarrow n \notin r$. Since $R^{\prime} \subseteq R$ by the definition of subhypergraph, it remains true that $\forall r \in R^{\prime} r \neq r^{*} \Longrightarrow n \notin r$; and so $r$ is a leaf of $H^{\prime}$ as well.

Proof of 4.28. In the following, let $s=\left(e_{1}, e_{2}, \ldots\right)$ be the seam sewn in the final operation. First, we must establish that $G^{\prime}$ is a sub-design of $G$. Since $f$ is not attached in $\sigma_{k-1}$ and since $\sigma_{k-1}$ is reachable, then by Lem.4.12, there cannot be any sewn edges in $\sigma_{k-1}$ adjacent to $f$. Since $E=\epsilon_{k}=\epsilon_{k-1} \cup\left\{e_{1}, e_{2}, \ldots\right\}$, the only edges adjacent to $f$ are the edges of $s$. All of the interior of $s$ must lie internal to $G$, so plucking $f$ will not 
disconnect $G$; $G^{\prime}$ is a well-defined sub-design. Furthermore, the internal edges of $G^{\prime}$ must be $\epsilon_{k-1}$, since plucking $f$ turns $e_{1}, e_{2}, \ldots$ into boundary edges, but cannot affect edges it is not adjacent to. Therefore, the set of faces and internal edges in $G^{\prime}$ is precisely the sets of $\sigma_{k-1}$; the prefix-plan is complete for $G^{\prime}$.

Proof of 4.29. We consider the case where $s$ is simple first, and then the case where $s$ is complex. If $s$ is simple and only one of the two adjacent faces $f_{1}$ (W.L.O.G.) is not covered by another hyperedge, then we are done. So suppose the other adjacent face $f_{2}$ is also not covered by another hyperedge. Then, by the definition of dual hyperedges, $s$ must be the only seam $f_{1}$ and $f_{2}$ are adjacent to. But by the hypothesis that $G$ has 3 or more faces, and by the well-formedness condition (Def.4.1) that the interior of $G$ is connected this cannot be the case. Therefore if $f_{1}=n^{*}$ is only covered by $r(s), f_{2}$ must be adjacent to another seam and thus covered by some other hyperedge.

If $s$ is complex, then we argue that every face $f$ adjacent to $s$ other than $f^{*}=n^{*}$ the common face, is covered by some hyperedge other than $r(s)$ regardless of whether $r(s)$ is a leaf-which suffices to show our desired result. Since $s=\left(e_{1}, \ldots, e_{k}\right)$ is a complex seam, by Lem.4.15 we know that all of the internal vertices $v_{1}, \ldots, v_{k-1}$ in the seam are T-junctions, with all stem-edges extending to the opposite side of $s$ from $f^{*}$ the common face. Let $e_{1,1}=e_{1}, e_{1,2}, \ldots, e_{1, l}=e_{2}$ be the edges radially ordered around $v_{1}$ and similarly $e_{i, j}$ the edges radially ordered around $v_{i}$. Let $f_{i}$ be the face adjacent to $e_{i}$ and opposite $f^{*}$. Observe that none of the $e_{i, j}$ that are not edges of $s$ can be colinear with each other or with $s$. Therefore there is a distinct maximal seam $s^{\prime}\left(e_{i, j}\right)$ associated to that edge, and a distinct dual hyperedge $r\left(s^{\prime}\left(e_{i, j}\right)\right)$ covering faces adjacent to that edge $e_{i, j}$. Since $e_{i, 2}$ and $e_{i, l-1}$ cannot be part of $s$, (a T-junction must have degree 3 or greater) and since at least one of those edges are adjacent to each of $f_{1}, \ldots f_{k}$, we have found the other dual hyperedges covering every node of $r(s)$ other than $n^{*}=f^{*}$. $\square$

Proof of Main Theorem 4.27. $\Rightarrow$ : Given that a design $G$ is foundation paper pieceable, we will show that its dual hypergraph $H_{G}$ is acyclic. We argue by induction on the number of faces in $G$. For the base case of induction, if $G$ has exactly two faces and is paper pieceable, then it has a single simple seam between the faces, and the associated hypergraph is a single binary hyperedge, which is trivially acyclic. For the inductive case, we may assume that for any pieceable sub-design $G^{\prime}$ of $G$, its dual hypergraph $H_{G^{\prime}}^{\prime}$ is acyclic.

In particular, let $G^{*}$ be the sub-design of $G=(V, F, x)$ defined as follows. Since $G$ is paper pieceable, there exists some plan $\sigma_{0} \rightarrow \cdots \rightarrow \sigma_{k-1} \rightarrow \sigma_{k}$ ending with the operation $O p_{k-1}^{1}\left(s^{*}\right): \sigma_{k-1} \rightarrow \sigma_{k}$. Let $\sigma_{k-1}=\left(\psi^{*}, \epsilon^{*}\right)$ be the penultimate state, s.t. $F=\psi^{*} \cup\left\{f^{*}\right\}$ and $f^{*} \notin \psi^{*}$. By Lem.4.28, $G^{*}$ the sub-design induced by $\psi^{*}$ is well-defined and $\sigma_{0} \rightarrow \cdots \rightarrow \sigma_{k-1}$ is a complete plan for $G^{*}$; hence it is foundation paper pieceable. By the inductive hypothesis, the dual hypergraph $H_{G^{*}}^{*}$ is acyclic. Above, we constructed $H_{G^{*}}^{*}$ via the sub-design $G^{*}$ induced by $\psi^{*} \subseteq F$. However, if we can show that $r\left(s^{*}\right)$ is a leaf hyperedge of $H_{G}$ and that $f^{*}$ is the corresponding unique leafnode (Lem.4.29), then plucking $r\left(s^{*}\right)$ from $H_{G}$ will induce a sub-hypergraph with $N^{\prime}=\psi^{*}=N^{*}$, which is simply $H_{G^{*}}^{*}$. By the inductive definition of acyclicity, this will suffice to show that $H_{G}$ is acyclic; our desired result.

So, we need simply show that $r\left(s^{*}\right)$ is a leaf hyperedge with leaf-node $f^{*}$. We claim that $s^{*}$ is the only maximal seam adjacent to $f^{*}$, since, if not $\sigma_{k-1}$ must have some edge $e$ which is sewn, but also adjacent to $f^{*}$, which is unattached, violating Lem.4.12. Since $s^{*}$ is the only maximal seam adjacent to $f^{*}, r\left(s^{*}\right)$ is the only hyperedge covering it, and $r\left(s^{*}\right)$ is a leaf hyperedge as desired.

$\Leftarrow$ : Let $G$ be a design with dual hypergraph $H_{G}$. We now show that if $H_{G}$ is acyclic, then $G$ is foundation paper pieceable. We again argue by induction on the number of faces in $G$. For the base case of induction, $G$ again has two faces, which must be connected with a single edge (single maximal simple seam) between them. Therefore, we may simply apply a
Type- 0 sewing operation. For the inductive case, we may assume that for any sub-design $G^{\prime}$ of $G$, if its associated hypergraph $H_{G^{\prime}}^{\prime}$ is acyclic, then $G^{\prime}$ is paper pieceable.

Since we are proving the converse direction of the theorem, we start by assuming that $H_{G}$ is acyclic, and derive from there that $G$ must be paper pieceable. We know that $H_{G}$ (being acyclic) must have some leaf hyperedge $r^{*}\left(s^{*}\right)$ (by Def.4.24). Furthermore by Lem.4.29, there is some $f^{*}=n^{*} \in r^{*}\left(s^{*}\right)$ s.t. $n^{*}$ is the unique node covered only by $r^{*}\left(s^{*}\right)$, and if $s^{*}$ is complex, that $f^{*}$ is the common face along $s^{*}$. By this construction, there cannot be any edge $e \notin s^{*}=\left(e_{1}, \ldots, e_{n}\right)$ adjacent to $f^{*}$. If there was, then $n^{*}=f^{*}$ would have to be covered by some other maximal seam that $e$ is a part of. Therefore the sub-design $G^{*}$ of $G$, induced by $F-\left\{f^{*}\right\}$ (where $F$ is the faces of $G$ ) is well-defined with internal edges $E^{*}=E-\left\{e_{1}, \ldots, e_{n}\right\}$ : $G^{*}$ is connected, and has all of the same maximal seams as $G$, less $s^{*}$. For this reason, the dual of this sub-design $H_{G^{*}}^{*}$ is the same as the hypergraph resulting from plucking $r^{*}\left(s^{*}\right)$ from $H_{G}$; and by the inductive definition of acyclicity, it must be acyclic as well. By the inductive hypothesis, and since $H_{G^{*}}^{*}$ is acyclic, $G^{*}$ must be paper pieceable; that is there must exist a plan $\sigma_{0} \rightarrow \cdots \rightarrow \sigma_{k-1}$. Since $G^{*}$ is a sub-design of $G$, this sequence of operations is also applicable to $G$, but leaves $f^{*}$ unattached and $s^{*}$ unsewn. The plan may be extended with one final operation $O p^{1}\left(s^{*}\right): \sigma_{k-1} \rightarrow \sigma_{k}$ that sews $s^{*}$ and attaches $f^{*}$. Therefore $G$ is also foundation paper pieceable. 